Max-Planck-Institut für demografische Forschung

Max Planck Institute for Demographic Research

Konrad-Zuse-Strasse 1 - D-18057 Rostock · GERMANY

Tel +49 (0) 3812081 - 0; Fax +49 (0) 3812081 - 202;

http://www.demogr.mpg.de

MPIDR WORKING PAPER WP 2005-001

FEBRUARY 2005

Nichteheliche Mutterschaft und soziale

Ungleichheit. Zur sozioökonomischen

Differenzierung der Familienformen

in Ost- und Westdeutschland

Dirk Konietzka (konietzka@ demogr.mpg.de)

Michaela Kreyenfeld (kreyenfeld@ demogr.mpg.de)

This working paper has been approved for release by: Andres Vikat (vikat@demogr.mpg.de)

Deputy Head of the Laboratory of Contemporary European Fertility and Family Dynamics.

(C) Copyright is held by the authors.

Working papers of the Max Planck Institute for Demographic Research receive only limited review. Views or opinions expressed in working papers are attributable to the authors and do not necessarily reflect those of the Institute. 


\title{
Nichteheliche Mutterschaft und soziale Ungleichheit Zur sozioökonomischen Differenzierung der Familienformen in Ost- und Westdeutschland
}

\author{
Dirk Konietzka und Michaela Kreyenfeld ${ }^{\star}$
}

Zusammenfassung: Nichteheliche Geburten haben in den 1990er Jahren in Westdeutschland, vor allem aber in Ostdeutschland stark an Bedeutung gewonnen. Der deutsche familialistische Wohlfahrtsstaat fördert jedoch steuerlich und sozialpolitisch vorrangig verheiratete Paare. Zugleich unterscheidet er zwischen Alleinerziehenden und nichtehelichen Lebensgemeinschaften. Vor diesem Hintergrund ist zu erwarten, dass verheiratete, kohabitierende und alleinerziehende Mütter sich in ihren sozialstrukturellen Hintergründen und hauptsächlichen Unterhaltsquellen (Einkommen des Partners, staatliche Transferzahlungen und eigenes Einkommen) unterscheiden. Analysen der Mikrozensen der Jahre 1991-2000 unterstützen diese These teilweise. Alleinerziehende Frauen haben häufiger einen geringen Bildungsabschluss und sind häufiger von sozialstaatlichen Transferzahlungen abhängig als Frauen in anderen Familienformen. In Westdeutschland leben gut ausgebildete Mütter vergleichsweise häufig in einer nichtehelichen Lebensgemeinschaft. Kohabitierende Mütter sind zudem häufiger Vollzeit erwerbstätig als andere Mütter. In Ostdeutschland bestehen nur geringe Unterschiede zwischen verheirateten und in einer nichtehelichen Lebensgemeinschaft lebenden Müttern im Hinblick auf den Bildungsabschluss und das Ausmaß der Erwerbsbeteiligung. Auch verheiratete Mütter sind selten vom Einkommen des Partners abhängig. Die ökonomische Unabhängigkeit verheirateter Mütter ist ein zentrales Merkmal des Wandels der Familie in Ostdeutschland und begründet deutliche Ost-West-Unterschiede in den ökonomischen Grundlagen von Familien.

Korrespondenzadresse: Max-Planck-Institut für demografische Forschung, Konrad-Zuse Str. 1, D18057 Rostock. Telefon: +49-381-2081-163 Email: konietzka@ demogr.mpg.de; kreyenfeld@demogr.mpg.de. 
English summary:

Non-marital motherhood and social inequality. Socio-economic differentiation of family forms in East and West Germany

Dirk Konietzka und Michaela Kreyenfeld

In the 1990s, non-marital motherhood has become more common in western and particularly in eastern Germany. The German familialistic welfare regime favours married couples, and, furthermore, treats single mothers differently from cohabiting mothers. Against this background, one would expect that married, cohabiting and single mothers differ by socio-economic characteristics and by the way they rely on partner income, transfers and own employment for their subsistence. Evidence from the German micro-census 1991 to 2000 gives partial support for this hypothesis. Single mothers are poorly educated and frequently rely on public transfers. In western Germany, mothers in non-marital unions are more often highly educated and employed full-time than married mothers. In eastern Germany, married mothers and those in marital unions do not differ by educational status nor by their employment behaviour. Even married mothers are rarely dependent on the partner income for their subsistence. The economic independence of married mothers is a central characteristic of family change in eastern Germany, and it contributes to persistent east-west-differences in the economic foundation of families. 


\section{Einleitung: Die Zunahme nichtehelicher Geburten und der Wandel der Familienformen}

Zu den auffälligsten Veränderungen des Familienbildungsprozesses in Deutschland im letzten Jahrzehnt gehört die Zunahme der nichtehelich geborenen Kinder. ${ }^{1}$ In den alten Bundesländern hat sich der Anteil nichtehelicher Geburten seit 1990 verdoppelt. Aktuellen Daten zu Folge fanden im Jahr 200321 Prozent aller Geburten außerhalb einer Ehe statt (Abbildung 1). Noch ausgeprägter ist der Wandel des Geburtenverhaltens in den neuen Bundesländern. Bereits in den 1950er Jahren lag der Anteil der nichtehelichen Geburten in der DDR höher als in der Bundesrepublik. Er stieg dann insbesondere in der zweiten Hälfte der 1970er und der ersten Hälfte der 1980er Jahre an und erreichte 1989 einen Höchststand von 33 Prozent. $^{2}$ Entgegen den Erwartungen hat nach dem Fall der Mauer und der Implementation des bundesrepublikanischen Institutionensystems der Anteil der nichtehelich geborenen Kinder in Ostdeutschland weiter zugenommen. Im Jahr 2003 wurden 57 Prozent aller Kinder außerhalb einer Ehe geboren, womit der Anteil nichtehelicher Geburten in den neuen Ländern gegenwärtig 36 Prozentpunkte höher als in den alten Ländern liegt. Diese Differenz hat im internationalen Vergleich ein bemerkenswertes Ausmaß. So befinden sich die neuen und die alten Bundesländer in Europa an den entgegengesetzten Enden eines Kontinuums (Council of Europe 2003). Nur in den südeuropäischen und einigen osteuropäischen Ländern ist die Nichtehelichenquote niedriger als in Westdeutschland.

1 Seit der Änderung des Kindschaftsrechts im Jahr 1998 wird in der amtlichen Statistik anstelle des Begriffs ,nichtehelich Geborene' der Ausdruck ,Kinder nicht miteinander verheirateter Eltern' verwendet. Der Einfachheit halber verwenden wir durchgängig den Begriff der nichtehelichen Geburten bzw. Geborenen. Es sei angemerkt, dass mit der Änderung des Kindschaftsrechts auch die Definition einer nichtehelichen Geburt leicht verändert wurde. Bis zur Reform des Kindschaftsrechts galt ein Kind als ehelich, solange es innerhalb von 302 Tagen nach Auflösung der Ehe geboren wurde. Seit der Reform des Kindschaftsrechts gelten Kinder, die nach Auflösung der Ehe geboren werden, als Kinder unverheirateter Eltern.

2 Als entscheidender Impuls für diesen Zuwachs galten sozialpolitische Maßnahmen - insbesondere die Einführung des ,Babyjahres', welches seit 1976 verheirateten Frauen ab dem zweiten Kind, unverheirateten Frauen aber bereits ab dem ersten Kind einen Anspruch auf einen einjährigen bezahlten Mutterschaftsurlaub einräumte (Gysi und Speigner 1983; Höhn 1992; Trappe 1995; Konietzka und Kreyenfeld 2002a, 2002b). Im Jahr 1986 wurde das Babyjahr auf alle Geburten, d.h. auch auf Erstgeburten innerhalb von Ehen ausgedehnt. 
Ostdeutschland liegt zusammen mit Schweden, Island und Estland am oberen Ende der europäischen Skala. ${ }^{3}$

Abbildung 1: Anteile nichtehelich Geborener an allen Geborenen in Ost- und Westdeutschland

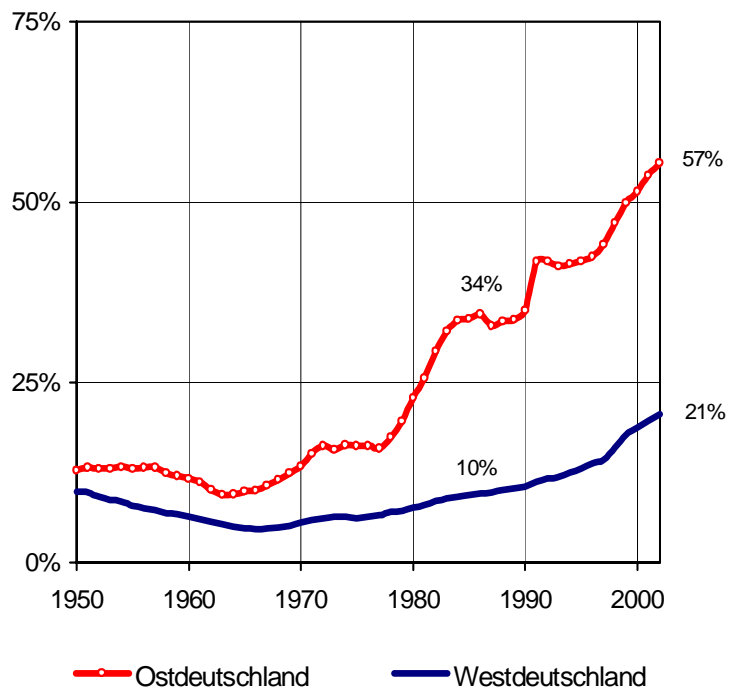

Anmerkung: Ab 2000 ohne Berlin.

Quelle: Statistisches Bundesamt (2001a). Die Werte für das Jahr 2001-2003 wurden uns vom Statistischen Bundesamt auf Anfrage bereit gestellt.

Das hohe und steigende Niveau der nichtehelichen Geburten in Ostdeutschland ist aus der Perspektive der Familienentwicklung auf eine Entkopplung von Familiengründung und Eheschließung in den Lebensverläufen von Frauen und Männern zurückzuführen. Hinter dem Phänomen nichtehelicher Elternschaft verbergen sich unterschiedliche, neue Familienformen', die auch in sozialstruktureller Hinsicht heterogen sind. Entsprechend können die Ursachen der Zunahme nichtehelicher Elternschaft variieren. Wir konzentrieren uns in diesem Beitrag auf die

3 Die Anteile nichtehelich Geborener lagen 2001 in Finnland bei 40\%, in Dänemark bei $45 \%$, in Norwegen bei $50 \%$, in Schweden bei $56 \%$ und in Island bei $63 \%$. Die niedrigsten Anteile in Europa wiesen Zypern mit 3\% und Griechenland mit 4\% auf. Große Unterschiede finden sich unter den mittel- und osteuropäischen Transformationsstaaten. Slowenien hatte im Jahr 2001 mit $39 \%$ einen viermal höheren Anteil nichtehelicher Geburten als Kroatien mit 9\%. Estland hatte einen Anteil von 56\%, Polen dagegen einen Anteil von 13\% (Council of Europe 2003). 
sozioökonomischen Hintergründe nichtehelicher Mutterschaft. ${ }^{4}$ Zwei Thesen lassen sich einander gegenüberstellen, die geradezu gegensätzliche sozialpolitische Implikationen aufweisen: auf der einen Seite die These sozialstaatlicher Ursachen nichtehelicher Mutterschaft und auf der anderen Seite die These der wachsenden ökonomischen Unabhängigkeit der Frau und ihrer Emanzipation von traditionellen Geschlechterrollen. ${ }^{5}$ Der ersten These zu Folge ist die Zunahme lediger Mutterschaft eine Folge spezifischer sozialpolitischer Anreize. Der in den USA und Großbritannien verbreiteten Sichtweise sind unverheiratete Mütter überwiegend jung (vielfach im Teenager-Alter); sie weisen eine niedrige Erwerbsquote und einen hohen Grad an Sozialhilfeabhängigkeit auf (Kilkey und Bradshaw 1997; McLaughlin 1997; McLanahan 2000; Lichter et al. 2002; Sigle-Rushton und McLanahan 2002; Lichter et al. 2004). Insbesondere in den USA wird staatlichen Sozialleistungen ein , anti-marriage bias' zugeschrieben, demzufolge junge Frauen, die schlechte Arbeitsmarktchancen haben, die strategische Entscheidung treffen, bei Mutterschaft nicht zu heiraten und stattdessen staatliche Hilfsprogramme in Anspruch zu nehmen (Garfinkel et al. 2003; Moffitt et al. 1998; Moffitt 2000). In den USA wurden insbesondere dem AFDC-Programm (Aid to Families with Dependent Children) solche Anreizwirkungen unterstellt (Fein 2001).

Eine gänzlich andere Perspektive auf nichteheliche Mutterschaft setzt an der wachsenden ökonomischen Eigenständigkeit von Frauen an. Mit der ökonomischen Theorie der Familie (Becker 1993) lässt sich argumentieren, dass die verbesserte Position von Frauen auf dem Arbeitsmarkt einen Bedeutungs- und Funktionsverlust der Ehe als Instanz ökonomischer und sozialer Sicherung zur Folge hat. Mit der feministischen Wohlfahrtsstaatstheorie ist zudem darauf $\mathrm{zu}$ verweisen, dass für die ökonomische Unabhängigkeit von Frauen mit Kindern nicht zuletzt institutionelle Faktoren wie ein öffentliches Kinderbetreuungssystem sowie sozial- und steuerpolitische Steuerungsinstrumente eine ausschlaggebende Bedeutung haben (Lewis 1992; Orloff 1993; Ostner 1995; Sainsbury 1997).

4 Zum Aspekt der Vaterschaft siehe z.B. Tölke und Hank (2004).

5 Im sozialpolitischen Diskurs in Deutschland wird nichtehelicher Mutterschaft außerdem häufig als eine soziale Notlage diskutiert, die überwiegend durch Trennung, Scheidung oder Verwitwung entsteht und besondere sozialpolitische Unterstützung erfordert (siehe Abschnitt II.2). Im Zentrum unserer Aufmerksamkeit stehen jedoch die ledige Mutterschaft und die mit dieser verbundenen sozioökonomischen Differenzierungen. 
Die beiden Thesen eröffnen ganz unterschiedliche Perspektiven auf die Ursachen der Zunahme lediger Mutterschaft. Insbesondere deren Implikationen für die sozioökonomische Position nicht verheirateter Mütter und die daraus folgenden Muster sozialer Ungleichheit gehen weit auseinander. Es ist das Ziel dieses Beitrags zu untersuchen, ob ledige Mutterschaft in Ost- und Westdeutschland eher Ausdruck sozioökonomischer Problemlagen oder einer zunehmenden ökonomischen Unabhängigkeit von Frauen ist. Im Rahmen dieser Fragestellung spielt die Unterscheidung zwischen verschiedenen neuen Familienformen und damit zwischen Frauen, die mit Kindern in einer nichtehelichen Lebensgemeinschaft leben, und Alleinerziehenden eine zentrale Rolle. ${ }^{6}$

Die Analyse der sozialen Ungleichheit neuer Familienformen bedarf eines theoretischen Bezugsrahmens, der die Perspektiven der ökonomischen Unabhängigkeit von Frauen und der Entstehung einer ,Versorgungsklasse“ alleinerziehender Mütter gleichermaßen umspannt. Ein wichtiger Punkt ist in diesem Zusammenhang, dass die ökonomische Situation von Frauen mit Kindern nicht nur durch individuelle Ressourcen wie Bildung, sondern auch durch sozialpolitische Rahmenbedingungen bestimmt wird (Cherlin 2000: 399; Sigle-Rushton und McLanahan 2002: 431). Wir untersuchen in diesem Sinne zum einen den Einfluss von Bildungsressourcen und weiteren sozialstrukturellen Faktoren auf die Familienformen von Frauen mit Kindern unter den in Ost- und Westdeutschland jeweils gegebenen institutionellen Rahmenbedingungen. Zum anderen befassen wir uns mit den ökonomischen Existenzgrundlagen von Frauen in den unterschiedlichen Familienformen. In welchem Ausmaß sind Frauen mit Kindern ökonomisch unabhängig oder vom Partnereinkommen oder von staatlichen Transferleistungen abhängig? Besondere Aufmerksamkeit widmen wir der Frage, ob sich diese Zusammenhänge in Ost- und Westdeutschland unterscheiden.

Im Folgenden diskutieren wir zunächst die sozial- und familienpolitischen Rahmenbedingungen in Deutschland, indem wir auf Konzepte der vergleichenden Wohlfahrtsstaatsforschung zurückgreifen. Auf dieser Basis leiten wir die

6 Die Bezeichnung ,neue Familienformen' ist insofern irreführend, als unverheiratete Mutterschaft in vielen europäischen Ländern auch in der Vergangenheit verbreitet war und unterschiedliche Bedeutungen erfahren hatte (Mitterauer 1983). Wir greifen auf diesen Begriff zurück, da er sich in der Familiensoziologie im Kontext der Diskussion um den Wandel der Lebens- und Familienformen eingebürgert hat. 
Untersuchungshypothesen zu den sozioökonomischen Hintergründen der verschiedenen Familienformen ab (Teil 2). In Teil 3 beschreiben wir die Daten der Mikrozensen aus den Jahren 1991 bis 2000. Teil 4 beinhaltet die empirischen Analysen, die zum einen aus der Darstellung der sozialstrukturellen Determinanten lediger Mutterschaft und zum anderem aus einem Vergleich des Ausmaßes der Erwerbstätigkeit und des sozialstaatlichen Transferbezugs von Frauen in Abhängigkeit von der Familienform bestehen.

\section{Wohlfahrtsstaatliche Ursachen sozialer Ungleichheit der Familienformen}

Familien- und sozialpolitische Rahmenbedingungen nehmen Einfluss auf die Gestaltung der Lebensläufe von Frauen und Männern, deren Rollen bzw. Positionen auf dem Arbeitsmarkt und in der Familie. Staatliche Transferleistungen oder Regeln der Besteuerung, die bestimmte Familienmodelle mit steuerlichen Privilegien ausstatten und andere von solchen ausschließen, schaffen aus der Perspektive des Individuums systematische Anreize bei der Wahl der Familienform. Sozialpolitischen Regelungen können insofern Steuerungswirkungen in Bezug auf das Erwerbsverhalten, die Eheschließung und die Familiengründung zugeschrieben werden. Sie setzen jedoch nicht nur Anreize für individuelle Entscheidungen, sie prägen auch soziale Ungleichheiten und innerfamiliale Abhängigkeitsverhältnisse.

Wohlfahrtsstaatliche Rahmenbedingungen entscheiden nicht zuletzt darüber, in welchem Maß die ökonomischen Existenzgrundlagen und die Absicherung sozialer Risiken von Frauen von der Familienform abhängen. Sie haben auch maßgeblichen Einfluss darauf, ob Frauen nach der Geburt eines Kindes nur die Wahl zwischen der ökonomischen Abhängigkeit vom Ehepartner und der ökonomischen Abhängigkeit vom sozialen Sicherungssystem haben, oder ob ihnen weitere Optionen der Organisation von Familie offen stehen (Orloff 1993; Sainsbury 1997; Daly 2000). Verfügen Frauen über beschränkte Möglichkeiten der Erwerbstätigkeit und stellt die Ehe die hauptsächliche Institution der ökonomischen Absicherung für Frauen mit Kindern dar, dann ist unverheiratete Elternschaft mehr oder weniger zwangsläufig mit einer prekären 
ökonomischen Lebenslage verbunden. Diese Situation war bis in die 1960er Jahre hinein für alle westlichen Länder charakteristisch. Seitdem haben die Optionen der ökonomischen Eigenständigkeit von Frauen in den europäischen Wohlfahrtsstaaten zugenommen - allerdings in unterschiedlicher Art und Weise.

\subsection{Markt, Staat und Familie}

In der international vergleichenden Sozialpolitikforschung ist die Strukturierung sozialer Ungleichheit in der Familie und der Abhängigkeitsbeziehungen zwischen den Geschlechtern in den Kategorien von Markt, Staat und Familie diskutiert worden (Lewis 1992; Ostner 1995; Esping-Andersen 1999). Die Wohlfahrtsstaatsforschung war traditionell auf die Analyse des Spannungsverhältnisses der Basisinstitutionen Staat und Markt und deren ungleichheitsgenerierende bzw. stratifizierende Bedeutung gerichtet. Das Ausmaß, in dem marktproduzierte soziale Risiken sozialpolitisch verringert oder ausgeglichen und damit Individuen aus der Abhängigkeit vom Marktverhältnissen bzw. Markteinkommen herauslöst werden (,De-Kommodifizierung'), variiert demzufolge nicht nur im internationalen Vergleich, sondern kann in systematischer Weise auf wohlfahrtsstaatliche Politik oder gar umfassendere ,Regimelogiken` zurückgeführt werden (Esping-Andersen 1990).

Der traditionelle Fokus der Sozialpolitikforschung auf das Markt-Staat-Verhältnis ist v.a. im Anschluss an die feministische Kritik in Frage gestellt worden (Lewis 1992; Orloff 1993; Ostner 1995). Mit dem Konzept der De-Kommodifizierung werden dieser zu Folge vor allem die Lebenschancen und -risiken von Männern bzw. des männlichen Ernährers und marktbedingte Risiken abfedernde Arbeitnehmerrechte thematisiert. Letztere haben für Frauen, solange deren Lebenslage primär durch familiale Abhängigkeitsverhältnisse bestimmt wird und sie auf familiale Betreuungs- und Pflegearbeit verwiesen sind, nur den Charakter abgeleiteter Versorgungsansprüche (Lewis 1992; Lister 1994; Ostner 1995). Das Ausmaß der ökonomischen Abhängigkeit der Ehefrau vom männlichen Ernährer ist vor diesem Hintergrund als eine zweite grundlegende Strukturierungsdimension sozialer Anrechte und Risiken zu betrachten. Um den Umfang politischer Maßnahmen zur Herauslösung der Frau aus familialen 
Abhängigkeitsverhältnissen zu kennzeichnen, wurden die Kategorien ,Familialismus“ und ,De-Familialisierung ' in die sozialpolitische Diskussion eingeführt (Sainsbury 1996: 39; Esping-Andersen 1999: 51). Diese Erweiterung der auf die Markt-StaatDimension konzentrierten sozialpolitischen Debatte um eine zweite, analytisch unabhängige Familie-Staat-Dimension der Strukturierung sozialer Ungleichheit ist mittlerweile in den Kanon der international vergleichenden Sozialpolitikforschung eingegangen.

Die Unterscheidung der Institutionen Markt, Staat und Familie ist im Folgenden die Grundlage für eine Analyse der ökonomischen Lage bzw. des Ausmaßes der ökonomischen Unabhängigkeit von unverheirateten Müttern in Ost- und Westdeutschland. Wir untersuchen den Stellenwert, den die eigene Erwerbstätigkeit (und damit die Existenzsicherung über den Markt), die Abhängigkeit vom Erwerbseinkommen des Partners (und damit der Familialisierung der Lebenslage) sowie die Transferabhängigkeit (von staatlichen Instanzen) für Frauen in den unterschiedlichen Familienformen unter den sozialpolitischen Rahmenbedingungen in Deutschland haben.

\subsection{Die sozialpolitischen Rahmenbedingungen der Familie im deutschen Wohlfahrtsstaat}

Der deutsche Sozialstaat wird vielfach als ,modales Beispiel ${ }^{\text {‘ des }}$ kontinentaleuropäischen familialistischen Wohlfahrtsregimes bezeichnet (Gauthier 1996; Gornick et al. 1998; Esping-Andersen 1999: 85; Blossfeld und Drobnič 2001). ${ }^{7}$ In diesem nimmt die Familie eine besondere Stellung als Ort gesellschaftlicher Wohlfahrtsproduktion ein. Die sozialpolitischen Regelungen unterstützen den als bürgerliche Familie bezeichneten Typus der Familie, d.h. insbesondere die Lebensform der Ehe und darin eine ausgeprägte geschlechtsspezifische Arbeitsteilung oder auch die 
,standard male bread-winner family“ (Esping-Andersen 1999: 83). Der Wandel der Lebens- und Familienformen, der Lebensläufe und Erwerbsmuster insbesondere der Frauen in den letzten Jahrzehnten ist zwar von der deutschen Sozialpolitik in der einen oder anderen Weise berücksichtigt worden. Das familienpolitische Leitbild ist jedoch nur partiell - in die Richtung eines modernisierten Versorgermodells mit der Teilzeitbzw. diskontinuerlichen Beschäftigung von Müttern - modifiziert worden (PfauEffinger 1998). Die Ehe als Lebensform besitzt nach wie vor einen privilegierten Stellenwert, was an einer Reihe sozial- und familienpolitischer Maßnahmen festgemacht werden kann.

\section{Ehe und Familie im Steuer- und Transfersystem}

Die grundgesetzliche Vorgabe, dass die Ehe unter dem besonderen Schutz des Staates steht (Artikel 6, § 1), findet im Steuer- und Sozialversicherungssystem in vielerlei Hinsicht ihren Niederschlag. Transferleistungen und Steuersubventionen fördern die Ehe vor allem als Institution geschlechtlicher Arbeitsteilung (Dingeldey 2001, 2002; Gerhard 2003). Instrumente wie die gemeinsame Veranlagung der Einkommenssteuer verschaffen aufgrund des progressiven Steuertarifs Ehepaaren die relativ größten Steuervorteile, wenn beide Partner sehr ungleiche Einkommen erzielen, bzw. einer der beiden nicht, geringfügig oder Teilzeit erwerbstätig ist. Wenn beide Ehepartner mehr oder weniger kontinuierlich Vollzeit erwerbstätig sind, fallen die finanziellen und versicherungstechnischen Vorteile der Ehe gering aus. Im Sozialversicherungssystem erhalten verheiratete Paare indirekte Transferleistungen in Form der Mitversicherung der nichterwerbstätigen Haushaltsmitglieder in der gesetzlichen Krankenversicherung und in Form der Hinterbliebenenrente in der Rentenversicherung. Aus dem für die Sozial- und Familienpolitik in Deutschland grundlegenden Subsidiaritätsprinzip wird abgeleitet, dass die Familie ihre Angelegenheiten in größtmöglichem Ausmaß eigenständig und selbstverantwortlich, ohne staatlichen Eingriff regeln soll (Sachße 2003). Mit dem Subsidiaritätsprinzip ist ebenfalls das sozialpolitische Ziel verbunden,

7 Dieser wird häufig auch mit einem konservativen oder katholischen Wohlfahrtsstaatsmodell identifiziert. Der Einschätzung, dass dieses Modell durch die katholische Soziallehre geprägt wurde, ist wiederum von Manow (2002: 203ff.) widersprochen worden. Für unsere Argumentationsführung ist die Diskussion über den konservativen Charakter oder auch den Stellenwert einer konfessionellen Konfliktlinie für die Entwicklung des deutschen Sozialstaates indes nicht von entscheidender Bedeutung. 
Familien „mit Blick auf besondere strukturelle Schwächen spezielle Hilfen“ (Wingen 1997: 119) anzubieten. Verschiedene Transferleistungen sollen Defizite in der individuellen Leistungsfähigkeit von Familien kompensieren - insbesondere in dem Fall, dass der Familienernährer temporär oder dauerhaft ausfällt.

\section{Familienpolitik und unverheiratete Elternschaft.}

In der heutigen Bundesrepublik herrscht - nicht anders als einst in der DDR - die Vorstellung vor, dass der Status, nicht verheiratet mit Kind" auf eine sozialpolitisch interventionsbedürftige soziale Notlage verweist. Alleinstehenden Eltern stehen daher besondere Leistungen zu. In der Bundesrepublik haben aber im Unterschied zum System der DDR nicht generell unverheiratete Eltern einen bevorzugten Zugang zu sozialpolitischen Leistungen. Es wird explizit zwischen Alleinerziehenden und nichtehelichen Lebensgemeinschaften mit Kindern unterschieden. Eine Bevorzugung von ,eheähnlichen“ gegenüber ehelichen Lebensgemeinschaften soll ausdrücklich ausgeschlossen werden. Damit sich keine systematischen Vorteile zugunsten nichtehelicher Lebensgemeinschaften ergeben, wird beispielsweise das Einkommen eines im gemeinsamen Haushalt lebenden Lebenspartners zur Bemessung des Anspruchs auf Sozialleistungen mit veranschlagt. Nicht berücksichtigt wird dagegen das Einkommen eines nicht im Haushalt lebenden Partners, weshalb Alleinerziehende leichter Zugang zu einkommens- und bedarfsabhängigen Transferleistungen wie Sozialhilfe, Wohngeld und Erziehungsgeld haben. Alleinerziehende gelten überdies als nur bedingt dem Arbeitsmarkt verfügbar, solange sie ein Kind unter 16 Jahren zu betreuen haben.

\section{Vereinbarkeit von Kind und Beruf}

Neben den steuer- und transferpolitischen Maßnahmen haben die Rahmenbedingungen der Vereinbarkeit von Kind und Beruf einen Einfluss auf die Organisationsform der Familie und damit auf die ökonomische Eigenständigkeit von Müttern. Die Betreuungsinfrastruktur als Basis der Vereinbarkeit von Kind und Beruf ist in Ost- und Westdeutschland sehr unterschiedlich ausgebaut. In Westdeutschland ist das Angebot an öffentlichen Betreuungsplätzen im Krippen- und Hortbereich seit den 1970er Jahren unverändert niedrig geblieben (siehe Tabelle 1 für die Versorgungsquoten mit Krippen-, 
Kindergarten- und Hortplätzen für die Jahre 1990 bis 2002). So liegt die Versorgungsquote im Krippenbereich unter 5 Prozent, im Hortbereich bei etwa 5 Prozent. In Ostdeutschland ist zwar im Krippenbereich die Versorgung mit Betreuungsplätzen von über 50 Prozent im Jahr 1990 auf unter 40 Prozent im Jahre 1994 zurückgegangen. Die Betreuungssituation ist dennoch deutlich besser geblieben als in Westdeutschland (Hank et al. 2004; Kreyenfeld 2003). Die Versorgungsquote im Krippenbereich lag 2002 um ein 10-faches höher als in den alten Bundesländern. ${ }^{8}$ Im Kindergartenbereich ist die Versorgungsquote im Westen erkennbar angestiegen, jedoch bestehen auch hier gravierende Ost-West-Unterschiede, da fast alle Betreuungsplätze in Westdeutschland Halbtagsbetreuungsplätze und im Osten Ganztagsbetreuungsplätze sind (Büchel und Spieß 2002; Deutsches Jugendinstitut 2002).

Tabelle 1: Anteil der Plätze in Kindertageseinrichtungen nach Anzahl der Kinder der jeweiligen Altersgruppe (Versorgungsquoten)

\begin{tabular}{|l|rrrr|rrrr|}
\hline & \multicolumn{4}{|c|}{ Westdeutschland } & \multicolumn{4}{c|}{ Ostdeutschland } \\
& 1990 & 1994 & 1998 & $2002^{*}$ & 1990 & 1994 & 1998 & $2002^{*}$ \\
\hline Krippe & $2 \%$ & $2 \%$ & $3 \%$ & $3 \%$ & $56 \%$ & $41 \%$ & $37 \%$ & $37 \%$ \\
Kindergarten & $78 \%$ & $85 \%$ & $102 \%$ & $103 \%$ & $113 \%$ & $117 \%$ & $132 \%$ & $121 \%$ \\
Hort & $5 \%$ & $5 \%$ & $6 \%$ & $6 \%$ & $88 \%$ & $34 \%$ & $48 \%$ & $59 \%$ \\
\hline
\end{tabular}

Anmerkung: Die Versorgungsquote berechnet sich aus der Anzahl der Plätze pro 100 Kinder der jeweiligen Altersgruppe. Für die Berechnung der Versorgungsquote für die Krippe wurden die Altersgruppe der 0- bis unter 3-Jährigen, für den Kindergarten die Altersgruppe der 3- bis unter 6Jährigen und für den Hort die Altersgruppe der 6- bis unter 10-Jährigen zugrunde gelegt.

*) ohne Berlin

Quelle: Deutsches Jugendinstitut, 1993; Statistisches Bundesamt (1996; 2001b; 2004)

\subsection{Die soziale Differenzierung der Familienformen im deutschen Wohlfahrtsstaat}

In der Summe fördern die sozial- und familienpolitischen Maßnahmen insbesondere das sogenannte bürgerliche Familienmodell in seiner traditionellen oder teilmodernisierten

8 Im Hortbereich suggeriert die Kinder- und Jugendhilfe-Statistik einen drastischen Abbau der Betreuungskapazitäten zwischen 1990 und 1994. Da jedoch Betreuungsplätze in Schulhorten nur teilweise in der amtlichen Statistik erfasst werden, sind diese Angaben nur bedingt aussagefähig (für eine Korrektur der amtlichen Daten, siehe DJI 2002). 
Variante. Die grundgesetzlich verankerten Normen zum Schutz der Ehe und die daraus abgeleiteten familienpolitischen Maßnahmen stabilisieren die Institution der Ehe in vielfacher Hinsicht. Sie setzen aus der individuellen Perspektive ökonomische Anreize zugunsten der Ehe und gegen andere Lebens- bzw. Familienformen. In Westdeutschland wird durch ein rudimentär ausgebautes Angebot institutioneller Kinderbetreuung überdies faktisch das Modell einer geschlechtsspezifischen Rollendifferenzierung in der Familie durchgesetzt.

Das Profil nichtehelicher Mutterschaft sollte unter den Bedingungen des deutschen Sozialstaates sozial heterogen sein. Das in der angloamerikanischen Diskussion vorherrschende Bild lediger Mütter als einer sozioökonomisch deprivierten, vielfach von sozialstaatlichen Transferzahlungen abhängigen Gruppe dürfte zumindest unvollständig sein. Da in Deutschland sozial- und familienpolitisch explizit zwischen Alleinerziehenden und nichtehelichen Lebensgemeinschaften differenziert wird, sind sozialstrukturelle Unterschiede zwischen alleinerziehenden und kohabitierenden Müttern zu erwarten. Die Entscheidung für eine nichteheliche Lebensgemeinschaft als Familienform hat nur dann keine negativen ökonomischen Folgen, wenn beide Partner nach der Familiengründung ihre ökonomische Unabhängigkeit aufrechterhalten. Die institutionellen Voraussetzungen für diese Familienform scheinen jedoch fast ausschließlich in Ostdeutschland gegeben.

Blickt man auf den empirischen Forschungsstand zum Wandel der Familienformen in Deutschland, dann lassen sich die genannten Annahmen über die Struktur der Familie in einem familialistischen Wohlfahrtsstaat insgesamt gut bestätigen. Verschiedene Studien haben gezeigt, dass die Entwicklung der familialen Lebensformen in der Bundesrepublik auch in den 1980er und frühen 1990er Jahren durch eine beträchtliche ,Strukturstarre' (Strohmeier 1993) geprägt wurde, d.h. die Versorgerehe das bei weitem dominante Familienmodell geblieben ist. Die These einer Pluralisierung der Familienformen in Westdeutschland ist von der Familienforschung über alle theoretischen Differenzen hinweg - zurückgewiesen worden (Meyer 1993; Höhn und Dorbritz 1995; Kaufmann et al. 1997; Nave-Herz 1997; Huinink und Wagner 1998). Die Frage einer Pluralisierung nichtfamilialer Lebensformen ist dagegen weniger eindeutig beantwortet worden (Strohmeier 1993; Diewald und Wehner 1996; Klein 1999; Wagner und Franzmann 2000). Eine zunehmende Pluralität partnerschaftlicher Lebensformen scheint sich insbesondere im Längsschnitt des Lebenslaufs 
herauszukristallisieren (Brüderl und Klein 2003). Insgesamt sprechen die empirischen Befunde dafür, dass die institutionellen Merkmale und Regulierungsprinzipien der Familie, die Definition und Gewährung sozialer Anrechte für Individuen und deren Familienangehörige die Verhaltensmuster bzw. Entscheidungen über die Familienform in Westdeutschland in hohem Maß geprägt haben. Die in der Einleitung präsentierten Daten zur Entwicklung der Anteile nichtehelich geborener Kinder verweisen jedoch darauf, dass sich die Familienformen in den letzten Jahren verändert haben. Dies trifft vor allem auf Ostdeutschland zu (Rupp 1998; Huinink 1999; Konietzka und Kreyenfeld 2002a, 2002b; Huinink und Konietzka 2003). Allerdings sind die Anteile der nichtehelichen Geburten auch in Westdeutschland gestiegen. Sie haben sich zwischen 1990 und 2001 verdoppelt und einen Anteil von 20 Prozent erreicht. Die Frage nach der mit steigenden Anteilen nichtehelicher Mutterschaft verbundenen Differenzierung der Familienformen und Strukturierung sozialer Ungleichheit stellt sich damit nicht nur für Ostdeutschland.

\subsection{Hypothesen}

Unsere Untersuchungshypothesen richten sich vor allem auf den Zusammenhang der neuen Familienformen mit Mustern sozialer Ungleichheit. Wir formulieren zum einen Erwartungen über die sozialstrukturellen Hintergründe von Frauen, die nach der Geburt eines Kindes in einer nichtehelichen Lebensgemeinschaft leben oder alleinerziehend sind. Zum anderen treffen wir Annahmen über die mit der Familienform einhergehenden Unterschiede im Ausmaß der Erwerbsbeteiligung und ökonomischen (Un-)Abhängigkeit von Müttern in der frühen Familienphase.

\section{Die These der ökonomischen Unabhängigkeit der Frau}

Unseren Überlegungen folgend hat die ökonomische Unabhängigkeit von Frauen eine grundlegende Bedeutung für die Wahl der Familienform. Wir gehen davon aus, dass mit zunehmenden Arbeitsmarktchancen von Frauen die Institution der Ehe im Zusammenhang mit der Familiengründung wichtige Funktionen verliert, insbesondere sich deren ökonomischer Nutzen reduziert. Angesichts der deutlichen Bevorteilung der Ehe im Steuer- und Transfersystem und der schlechten Vereinbarkeitsbedingungen von 
Familie und Erwerbsarbeit wird die Entscheidung für eine nichteheliche Lebensgemeinschaft als Familienform in Westdeutschland in den meisten Fällen finanziell bestraft werden. Die nichteheliche Lebensgemeinschaft sollte daher am ehesten für gut ausgebildete Frauen mit entsprechend guten Erwerbschancen als Alternative zur Ehe in Frage kommen. In Ostdeutschland sind die Rahmenbedingungen der Vereinbarkeit weniger restriktiv, weshalb nichteheliche Lebensgemeinschaften dort sozialstrukturell weniger selektiv sein sollten (Hypothese la).

Entscheidend ist in diesem Zusammenhang das Argument, dass die nichteheliche Lebensgemeinschaft nur dann gegenüber der Ehe keine finanziellen Nachteile mit sich bringt, wenn ein Doppelversorger-Arrangement in der Familie realisiert wird, d.h. insbesondere Frauen nach der Geburt eines Kindes relativ frühzeitig in Vollzeiterwerbstätigkeit zurückkehren. Wir erwarten daher, dass Frauen, die in einer nichtehelichen Lebensgemeinschaft leben, die eigene Erwerbstätigkeit nach der Geburt eines Kindes nur kurzzeitig oder geringfügig einschränken (Hypothese 1 b).

\section{Die These der Sozialstaatsabhängigkeit lediger Mütter}

Legt man die in der internationalen Literatur vorherrschende Perspektive zu Grunde, dann hat die Gruppe der Alleinerziehenden einen sozial selektiven Charakter. Nach Friedman et al. (1994) kann für junge, schlecht ausgebildete Frauen Mutterschaft als biographische Alternativrolle angesichts unsicherer und unbefriedigender Erwerbsaussichten fungieren. Demnach ist für junge Frauen, die keinen oder einen niedrigen Schulabschluss besitzen und schlechte Erwerbsperspektiven haben, die Wahrscheinlichkeit alleinerziehender Mutterschaft überproportional hoch (Hypothese 2a). Hieran schließt die Erwartung an, dass die sozioökonomische Situation Alleinerziehender vielfach durch die Abhängigkeit von staatlichen Transferleistungen geprägt wird. Wie stark diese ausgeprägt ist, hängt wiederum von den sozialpolitischen Rahmenbedingungen ab. Die Chancen alleinerziehender Frauen, unabhängig von staatlichen Transferleistungen zu leben, dürften am besten sein, wenn die Arbeitsmarktchancen und Vereinbarkeitsbedingungen günstig sind. Zwar bietet der deutsche Sozialstaat Alleinerziehenden eine Reihe besonderer Unterstützungsmaßnahmen, darunter einen bevorzugten Zugang zu Kinderbetreuungsplätzen. Das in Westdeutschland geringe institutionelle 
Betreuungsangebot für Kleinkinder schränkt jedoch für die Mehrzahl alleinerziehender Mütter die Option ein, erwerbstätig zu sein bzw. zu bleiben. Vor diesem Hintergrund dürften sozialpolitische Transferleistungen für Alleinerziehende die wichtigste Einkommensquelle darstellen (Hypothese 2b). Die im Osten vergleichsweise gute Versorgung mit Ganztagsbetreuungsplätzen bietet Alleinerziehenden zwar bessere Möglichkeiten der Vereinbarkeit von Familie und Erwerbsleben. Diese werden jedoch durch eine schlechtere Arbeitsmarktlage und hohe Arbeitslosigkeit konterkariert, so dass auch in Ostdeutschland ein relatives hohes Maß der Abhängigkeit der Alleinerziehenden von staatlichen Transferzahlungen zu erwarten ist.

Wir können vor dem Hintergrund dieser Überlegungen den Familienformen in idealtypischer Weise unterschiedliche sozioökonomische Konstellationen zuordnen. In Abbildung 2 sind die familialen Erwerbs- bzw. Ernährermodelle, die hauptsächlichen Unterhaltsquellen sowie der Umfang der Erwerbsbeteiligung von Frauen in den verschiedenen Familienformen zusammengefasst. Die hypothetischen Differenzen zwischen Müttern in nichtehelichen Lebensgemeinschaften und Alleinerziehenden im Hinblick auf die Erwerbsbeteiligung und die hauptsächlichen Unterhaltsquellen haben wir bereits ausführlich diskutiert. Das typische Familienmodell von verheirateten Frauen in der frühen Familienphase sollte demgegenüber das männliche Versorgermodell sein, das seinerseits eine reduzierte Erwerbstätigkeit der Frau und erhöhte ökonomische Abhängigkeit vom Ehemann impliziert.

Abbildung 2: Die sozioökonomische Basis unterschiedlicher Familienformen (idealtypisches Schema)

\begin{tabular}{l|lll}
\hline & Ehe & NEL & Alleinerziehend \\
\hline Basisinstitution sozialer Sicherung & Familie & Markt & Staat \\
Familienmodell & Male breadwinner & Dual breadwinner & Welfare mother \\
Erwerbsbeteiligung der Frau & gering & hoch & gering \\
Unterhaltsquelle der Frau & Partnereinkommen & Eigenes Einkommen & Staatliche Transfers \\
\hline
\end{tabular}




\section{Daten und Vorgehensweise}

\subsection{Stichprobenauswahl}

Für die empirischen Analysen verwenden wir die Scientific-Use-Files der Mikrozensen der Jahre 1991, 1993, 1996, 1998 und 2000 (Schimpl-Neimanns 1998; Köhler et al. 2000). Der Mikrozensus ist eine Ein-Prozent-Stichprobe der in Deutschland lebenden Bevölkerung. Das Statistische Bundesamt stellt eine auf der Haushaltsebene gezogene 70-Prozent-Teilstichprobe der Originalstichprobe als ,Scientific-Use-File' bereit. Der Mikrozensus hat für Analysen der Lebens- und Familienformen gegenüber anderen Befragungsdatensätzen wie dem Sozio-Oekonomischen Panel und dem DJIFamiliensurvey den wichtigen Vorteil, dass er aufgrund seiner hohen Fallzahlen differenzierte Vergleiche für Ost- und Westdeutschland ermöglicht. Da der Mikrozensus seit dem Jahr 1996 explizite Informationen zum ,Lebensformentyp’ enthält, können auch unterschiedliche Lebens- und Familienformen differenziert werden.

Das Ziel des ersten Teils unserer empirischen Analysen ist es, die Determinanten der Familienformen, in denen Frauen unmittelbar nach der Geburt eines Kindes leben, näher zu bestimmen. Diese Fragestellung lässt sich nicht direkt bearbeiten, da im Mikrozensus weder die Partnerschafts- noch die Fertilitätsbiografie erhoben werden. Mit gewissen Einschränkungen kann die Fertilitätsgeschichte jedoch auf Basis des Haushalts- bzw. Familienzusammenhangs der Befragten rekonstruiert werden. Hierzu wählen wir für unsere Analysen zunächst Frauen im reproduktiven Alter (d.h. zwischen 16 und 45 Jahren) aus. Wir beschränken uns zudem auf Befragte, die in privaten Haushalten und mit einem 0- bis 1-jährigen Kind im selben Familienzusammenhang leben (die also ein Kind haben, das im Jahr der Befragung oder im Jahr zuvor geboren wurde). ${ }^{9}$ Geschiedene oder verwitwete Personen schließen wir aus den Analysen aus. Die verbleibende Stichprobe umfasst pro Jahr etwa 9.000 Befragte in Westdeutschland.

9 Ein Beispiel: Eine Frau ist als Partnerin der Bezugsperson in einer nichtehelichen Lebensgemeinschaft gekennzeichnet. Sie lebt mit einem Kind, das im Jahr der Befragung geboren wurde, im gleichen Familienzusammenhang. Auf Basis dieser Information ist der Rückschluss möglich, dass die Befragte im letzten Jahr Mutter geworden ist. Da die Partnerschaftsbiographie nicht im Mikrozensus erhoben wird, kann jedoch nicht bestimmt werden, ob die Befragte auch schon zum Zeitpunkt der Geburt des Kindes in einer nichtehelichen Lebensgemeinschaft gelebt hat. Für Frauen, die im Jahr der Befragung oder im Jahr davor ein Kind bekommen haben, nehmen wir also an, dass der aktuelle Lebensformenkontext mit dem zum Zeitpunkt der Geburt des Kindes übereinstimmt. 
Die ostdeutsche Stichprobe enthält für das Jahr 1991 2.500 Befragte. Aufgrund des Geburtenrückgangs nach 1989 ist die ostdeutsche Stichprobe mit etwa 1.000 Befragten in den folgenden Jahren deutlich kleiner (siehe Tabelle 2).

\subsection{Methode}

Mit Hilfe logistischer Regressionsmodelle bestimmen wir den Einfluss sozioökonomischer Merkmale auf die Wahrscheinlichkeit (bzw. den Logarithmus des Odds), in einer bestimmten Familienform zu leben. Da wir nur für die Jahre 1996 bis 2000 Information über nichteheliche Lebensgemeinschaften haben, unterteilen wir die entsprechenden Analysen in zwei Schritte. Im ersten Schritt, in dem wir die Mikrozensen der Jahre 1991 bis 2000 verwenden, unterscheiden wir nur danach, ob die befragte Person ledig oder verheiratet ist. Im zweiten Analyseschritt schätzen wir für die Jahre 1996 bis 2000, für die der Mikrozensus die Unterscheidung zwischen nichtehelichen Lebensgemeinschaften und Alleinerziehenden erlaubt, multinomiale logistische Regressionsmodelle der Wahrscheinlichkeit, dass eine Frau mit einem 0- bis 1-jährigen Kind alleinerziehend ist, in einer nichtehelichen oder aber einer ehelichen Lebensgemeinschaft lebt.

Im Mikrozensus werden nichteheliche Lebensgemeinschaften dadurch abgegrenzt, dass zunächst eine Bezugsperson im Haushalt festgelegt wird. Für die weiteren Personen im Haushalt wird erfragt, ob es sich um den Lebenspartner oder die Lebenspartnerin der zuvor festgelegten Bezugsperson handelt. Das Statistische Bundesamt stellt auf Basis dieser Informationen eine Variable zur Verfügung, welche die ,Stellung innerhalb der Lebensgemeinschaft" anzeigt. Darüber hinaus wird eine Variable generiert, die den Lebensformenkontext angibt, in welchem der bzw. die Befragte lebt (Heidenreich und Nöthen 2002; Stauder 2002). Auf dieser Grundlage haben wir Frauen in nichtehelichen Lebensgemeinschaften dadurch identifiziert, dass sie in einer nichtehelichen Lebensgemeinschaft leben und entweder als Bezugsperson oder als Partnerin (nicht aber als Kind) der Bezugsperson in der nichtehelichen Lebensgemeinschaft klassifiziert sind. 


\subsection{Unabhängige Variablen}

Eine zentrale unabhängige Variable unserer Analysen ist die Bildung der Frau. Wir ziehen den höchsten Schulabschluss heran, den wir in drei Kategorien eingeteilt haben. Die erste Kategorie umfasst Befragte, die einen Hauptschulabschluss oder keinen Schulabschluss haben. Da nur wenige Befragte keinen Schulabschluss haben, war es nicht sinnvoll, zwei separate Kategorien für Hauptschulabsolventinnen und Frauen ohne Abschluss zu konstruieren. Die zweite Gruppe umfasst Frauen mit einem Realschulabschluss oder einem Abschluss der Polytechnischen Oberschule (POS), die dritte Gruppe schließlich Frauen mit Fachhochschulreife oder Abitur. ${ }^{10}$ Während die Frage zum Schulabschluss in den Befragungen 1996 bis 2000 zum festen Frageprogramm des Mikrozensus gehörte und daher Auskunftspflicht bestand, war diese Frage in den früheren Jahren Teil des freiwilligen Frageprogramms. Aus diesem Grund lag der Anteil der Personen ohne Angabe zum Bildungsabschluss in Westdeutschland 1991 bzw. 1993 mit 12 bzw. 13 Prozent deutlicher höher als in den späteren Befragungen. ${ }^{11}$

Wir unterscheiden weiterhin nach der Kinderzahl, d.h. danach, ob eine Frau nur ein Kind oder bereits zwei oder mehr Kinder hat. Da die Fertilitätsbiografie nicht im Frageprogramm des Mikrozensus enthalten ist, leiten wir diese Information auf Basis der Anzahl der im Haushalt lebenden Kinder ab. Mit dem Alter der Frau steigt die Wahrscheinlichkeit, dass Kinder bereits den elterlichen Haushalt verlassen haben. Da unsere Stichprobe nur Frauen im Alter von 16 bis 45 Jahren umfasst, sind die Abweichungen von der tatsächlichen Kinderzahl gering (Kreyenfeld und Konietzka 2003).

10 In der DDR gab es eine Unterscheidung zwischen dem Abschluss der POS nach der 8. Klasse und nach der 10. Klasse. Wir können diese beiden Abschlüsse mit den Daten des Mikrozensus nicht unterscheiden. Jedoch waren die Anteile mit einem 8-jährigen Abschluss in den jüngeren Kohorten vergleichsweise gering. Beispielsweise hatten nur 8 Prozent der um 1960 geborenen Frauen die POS mit der 8. Klasse abgeschlossen (Huinink et al. 1995: 107).

11 Idealerweise würde man neben dem Schulabschluss den Berufsbildungs- bzw. Hochschulabschluss verwenden. Leider ist diese Variable nicht mit den gleichen Antwortvorgaben in den unterschiedlichen Jahren erhoben worden. Darüber hinaus ergeben sich sehr viele Ausfälle für das Jahr 1991, in dem der Ausbildungs- bzw. Hochschulabschluss Teil des freiwilligen Frageprogramms des Mikrozensus war. 
Eine weitere unabhängige Variable ist das Alter der Befragten. Wir verwenden das Alter als kategoriale Variable und unterscheiden Frauen im Alter von 16 bis 20, 21 bis 25,26 bis 30 und 31 bis 45 Jahren.

Die Nationalität der Befragten dient in erster Linie als Kontrollvariable. Wir haben die Angaben zur Nationalität dichotomisiert, d.h. wir unterscheiden Personen mit deutscher und mit anderer Staatsangehörigkeit. Während es im Jahr 1991 nicht möglich war, Personen mit doppelter Staatsangehörigkeit zu identifizieren, wurden in den Jahren 1996 bis 2000 Personen mit deutscher und einer weiteren Staatsangehörigkeit gesondert ausgewiesen. Personen mit doppelter Staatsangehörigkeit haben wir zu den Nichtdeutschen gezählt.

Als weitere Kontrollvariable verwenden wir die Gemeindegrößenklasse. Idealerweise würde man Informationen über die Herkunftsregion oder die Region, in der Personen vor der Heirat bzw. der Geburt des ersten Kindes gelebt haben, verwenden. Da die Herkunftsregion im Mikrozensus nicht abgefragt wird, ziehen wir die Gemeindegröße der Region zum Befragungszeitpunkt heran. Wir unterscheiden Gemeinden mit weniger als 20.000, mit 20.000 bis 100.000 und mit mehr als 100.000 Einwohnern. Die Klassifikation ist durch die Vergröberungen der Gemeindegrößenklassen, die das Statistische Bundesamt bei der Datenweitergabe vornimmt, weitgehend vorgegeben (Köhler et al. 2000). Für Gemeinden im Saarland, in Brandenburg und in Mecklenburg-Vorpommern erlaubt der Scientific-Use-File des Mikrozensus nicht, zwischen Großstädten (mit mehr als 100.000 Einwohnern) sowie mittelgroßen Städten und Gemeinden (mit 20.000 bis 100.000 Einwohnern) zu unterscheiden. Diese Städte bzw. Gemeinden haben wir den mittelgroßen Städten zugeordnet.

\section{Empirische Ergebnisse}

In Tabelle 2 ist die Verteilung der in der multivariaten Analyse verwendeten Variablen wiedergegeben. Die Stichprobe umfasst Frauen mit 0- bis 1-jährigen Kindern und gibt damit einen Überblick über deren Lebenszusammenhang im Jahr der bzw. nach der Geburt eines Kindes. Auf der Basis dieser Tabelle lassen sich Rückschlüsse ziehen, inwieweit der Anstieg der Nichtehelichenquote auf eine Zunahme alleinerziehender 
Mutterschaft oder die wachsende Bedeutung nichtehelicher Lebensgemeinschaften mit Kindern hindeutet.

Die Anteile lediger Mütter haben sich im Laufe der 1990er Jahre in West- und Ostdeutschland verdoppelt. 1991 waren in Westdeutschland 6 Prozent der Frauen mit 0bis 1-jährigen Kindern ledig. Der Anteil erhöhte sich auf 8 Prozent im Jahr 1996 und 12 Prozent im Jahr 2000. In Ostdeutschland sind die Anteile lediger Mütter zugleich von 23 Prozent im Jahr 1991 über 36 Prozent im Jahr 1996 auf 47 Prozent im Jahr 2000 gestiegen. Wie ebenfalls aus der Tabelle ersichtlich wird, ist in Westdeutschland die eine Hälfte der unverheirateten Mütter alleinerziehend und die andere Hälfte lebt in einer nichtehelichen Lebensgemeinschaft. In Ostdeutschland haben Frauen, die in einer nichtehelichen Lebensgemeinschaft leben, eine deutlich größere Bedeutung als Alleinerziehende. Im Jahr 2000 lebten 30 Prozent aller Mütter mit 0- bis 1-jährigen Kindern in einer nichtehelichen Lebensgemeinschaft und 17 Prozent waren alleinerziehend.

Aufgrund der unterschiedlichen Bildungssysteme der DDR und der Bundesrepublik sind die Bildungsverteilungen in Ost und West sehr verschieden. Während in Westdeutschland mehr als 35 Prozent der Frauen mit Kindern einen Hauptschulabschluss oder keinen Schulabschluss haben, befinden sich maximal 10 Prozent der ostdeutschen Frauen in dieser Kategorie. Dagegen ist der Anteil der Frauen mit Abitur in Westdeutschland höher als in Ostdeutschland. Die große Mehrzahl der Frauen in den neuen Bundesländern verfügt statt dessen über einen mittleren Bildungsabschluss (der Polytechnischen Oberschule oder der Realschule). Das formale Bildungsniveau ist damit insgesamt sehr homogen. Wir überschätzen in Ostdeutschland die Anteile mit einem mittleren Bildungsniveau etwas, da die MikrozensusKlassifikation eine Unterscheidung zwischen den Absolventen der POS mit 8. Klasse und mit 10. Klasse nicht zulässt. Der hieraus resultierende Fehler dürfte aber nicht sehr groß sein, da in der DDR nur ein relativ kleiner Teil der Frauen die POS mit einem Abschluss der 8. Klasse verließ (vgl. Fußnote 11).

In Ostdeutschland ist der Anteil der Frauen mit einem Kind im Lauf der 1990er Jahre von 45 auf 56 Prozent gestiegen. Hier zeigt sich sehr wahrscheinlich die verringerte ostdeutsche Zweitgeburtenrate (Kreyenfeld 2003). Zugleich ist der Anteil der Frauen unter 26 Jahre gesunken und der Anteil der über 30-jährigen Frauen 
gestiegen. Dennoch ist das Alter der Mütter in der ostdeutschen Stichprobe niedriger als in der westdeutschen Stichprobe. Hier spiegeln sich Ost-West-Unterschiede im Geburtenverhalten wider. Das Alter bei der Erstgeburt ist in Ostdeutschland im Lauf der 1990er Jahre rapide angestiegen, hat jedoch das hohe westdeutsche Altersniveau (noch) nicht erreicht (Kreyenfeld und Konietzka 2004). Schließlich sind die Anteile der nichtdeutschen und der in Großstädten über 100.000 Einwohner lebenden Frauen im Osten geringer.

Tabelle 2: $\quad$ Deskriptive Statistiken (Spaltenprozente)

\begin{tabular}{|c|c|c|c|c|c|c|c|c|c|c|}
\hline & \multicolumn{5}{|c|}{ Westdeutschland } & \multicolumn{5}{|c|}{ Ostdeutschland } \\
\hline & 1991 & 1993 & 1996 & 1998 & 2000 & 1991 & 1993 & 1996 & 1998 & 2000 \\
\hline \multicolumn{11}{|l|}{ Familienstand } \\
\hline Verheiratet & $94 \%$ & $93 \%$ & $92 \%$ & $90 \%$ & $88 \%$ & $77 \%$ & $72 \%$ & $64 \%$ & $61 \%$ & $53 \%$ \\
\hline Ledig & $6 \%$ & $7 \%$ & $8 \%$ & $10 \%$ & $12 \%$ & $23 \%$ & $28 \%$ & $36 \%$ & $39 \%$ & $47 \%$ \\
\hline \multicolumn{11}{|l|}{ Familienform } \\
\hline Verheiratet & -- & -- & $92 \%$ & $90 \%$ & $88 \%$ & -- & -- & $64 \%$ & $61 \%$ & $53 \%$ \\
\hline NEL & -- & -- & $4 \%$ & $5 \%$ & $7 \%$ & -- & -- & $23 \%$ & $25 \%$ & $30 \%$ \\
\hline Alleinerziehend & -- & -- & $4 \%$ & $4 \%$ & $5 \%$ & -- & -- & $14 \%$ & $15 \%$ & $17 \%$ \\
\hline \multicolumn{11}{|l|}{ Bildungsabschluss } \\
\hline Hauptschule/kein Abschluss & $38 \%$ & $37 \%$ & $40 \%$ & $37 \%$ & $36 \%$ & $6 \%$ & $5 \%$ & $7 \%$ & $9 \%$ & $10 \%$ \\
\hline Realschule/POS & $31 \%$ & $32 \%$ & $32 \%$ & $34 \%$ & $33 \%$ & $74 \%$ & $75 \%$ & $75 \%$ & $69 \%$ & $68 \%$ \\
\hline Abitur & $18 \%$ & $18 \%$ & $24 \%$ & $26 \%$ & $27 \%$ & $15 \%$ & $13 \%$ & $16 \%$ & $19 \%$ & $19 \%$ \\
\hline keine Angabe & $12 \%$ & $13 \%$ & $4 \%$ & $3 \%$ & $4 \%$ & $5 \%$ & $6 \%$ & $2 \%$ & $2 \%$ & $2 \%$ \\
\hline \multicolumn{11}{|l|}{ Anzahl der Kinder } \\
\hline Ein Kind & $46 \%$ & $47 \%$ & $47 \%$ & $46 \%$ & $45 \%$ & $45 \%$ & $48 \%$ & $48 \%$ & $49 \%$ & $56 \%$ \\
\hline Zwei oder mehr Kinder & $54 \%$ & $53 \%$ & $53 \%$ & $54 \%$ & $55 \%$ & $55 \%$ & $52 \%$ & $52 \%$ & $51 \%$ & $44 \%$ \\
\hline \multicolumn{11}{|l|}{ Alter } \\
\hline $16-20$ & $3 \%$ & $3 \%$ & $2 \%$ & $2 \%$ & $2 \%$ & $7 \%$ & $5 \%$ & $4 \%$ & $5 \%$ & $5 \%$ \\
\hline $21-25$ & $19 \%$ & $18 \%$ & $15 \%$ & $14 \%$ & $14 \%$ & $39 \%$ & $38 \%$ & $25 \%$ & $22 \%$ & $23 \%$ \\
\hline $26-30$ & $42 \%$ & $41 \%$ & $36 \%$ & $34 \%$ & $30 \%$ & $37 \%$ & $39 \%$ & $44 \%$ & $40 \%$ & $37 \%$ \\
\hline $31-45$ & $37 \%$ & $38 \%$ & $46 \%$ & $51 \%$ & $54 \%$ & $17 \%$ & $18 \%$ & $26 \%$ & $33 \%$ & $35 \%$ \\
\hline \multicolumn{11}{|l|}{ Nationalität } \\
\hline Deutsch & $86 \%$ & $85 \%$ & $84 \%$ & $83 \%$ & $83 \%$ & $99 \%$ & $98 \%$ & $98 \%$ & $98 \%$ & $97 \%$ \\
\hline Andere & $14 \%$ & $15 \%$ & $16 \%$ & $17 \%$ & $17 \%$ & $1 \%$ & $2 \%$ & $2 \%$ & $2 \%$ & $3 \%$ \\
\hline \multicolumn{11}{|l|}{ Gemeindegröße } \\
\hline 0-20.000 Einwohner & $44 \%$ & $44 \%$ & $43 \%$ & $43 \%$ & $43 \%$ & $51 \%$ & $48 \%$ & $48 \%$ & $52 \%$ & $50 \%$ \\
\hline $20.000-100.000$ E. & $40 \%$ & $39 \%$ & $42 \%$ & $42 \%$ & $42 \%$ & $39 \%$ & $41 \%$ & $41 \%$ & $38 \%$ & $42 \%$ \\
\hline 100.000 u. m. E. & $15 \%$ & $17 \%$ & $15 \%$ & $15 \%$ & $15 \%$ & $10 \%$ & $11 \%$ & $11 \%$ & $10 \%$ & $8 \%$ \\
\hline Fallzahlen & 5.619 & 5.308 & 4.969 & 5.273 & 4.994 & 1.202 & 616 & 597 & 684 & 784 \\
\hline
\end{tabular}

Anmerkung: Die Stichproben umfassen Frauen, die zum Befragungszeitpunkt zwischen 16 und 45 Jahre alt sind und Kinder zwischen 0 und 1 Jahren alt haben, die im selben Haushalt leben.

Quelle: Scientitic-Use-File des Mikrozensus 1991, 1993, 1996, 1998, 2000 (eigene Berechnungen) 


\subsection{Sozialstrukturelle Determinanten lediger Mutterschaft 1990-2000}

Im ersten Teil unserer Analysen untersuchen wir die sozialstrukturellen Determinanten lediger Mutterschaft. Wie ausgeführt, unterscheiden wir zwei Hypothesen über die Bedeutung der Bildungsressourcen für die Familienform. Die erste Hypothese besagt, dass mit höherer Bildung der Frau der Stellenwert der Ehe im Zusammenhang mit der Familiengründung sinkt. Der zweiten Annahme zu Folge bleiben dagegen Frauen mit geringem Bildungsabschluss mit besonders großer Wahrscheinlichkeit nach der Geburt eines Kindes unverheiratet. Diese beiden zentralen Hypothesen sollen im Folgenden untersucht überprüft werden.

Wir beginnen auf der Basis der Mikrozensen der Jahre 1991 bis 2000 mit einer Analyse der Einflussfaktoren auf die Wahrscheinlichkeit, dass eine Frau, die im Jahr der Befragung bzw. im Jahr davor ein Kind bekommen hat, ledig ist. ${ }^{12}$ In Tabelle 3 sind die Ergebnisse der logistischer Regressionsmodelle wiedergegeben. Modell 1 zeigt erwartungsgemäß große Ost-West-Differenzen im Hinblick auf die Wahrscheinlichkeit, dass eine Frau nach der Geburt eines Kindes unverheiratet ist. Weiterhin ist ein klarer Anstieg lediger Mutterschaft über die Befragungsjahre festzustellen. Dieser Zusammenhang besteht unberührt von anderen Faktoren. Wie Modell 2 zeigt, sind Frauen mit mehr als einem Kind deutlich seltener ledig als Frauen, die zum ersten Mal Mutter geworden sind. Ferner besteht ein klarer Zusammenhang zwischen dem Alter der Frau und ihrem Familienstand. Eine Mutterschaft im Alter von unter 21 Jahren erhöht die Odds, ledig zu sein. Allerdings hat frühe Mutterschaft nach wie vor eine vergleichsweise geringe quantitative Bedeutung. Nur zwei bis drei Prozent der Frauen in Westdeutschland und fünf Prozent der Frauen in Ostdeutschland mit 0- bis 1-jährigen Kindern sind jünger als 21 Jahre (siehe Tabelle 2). Schließlich ist Modell $3 \mathrm{zu}$ entnehmen, dass ausländische Frauen mit einer sehr viel geringeren Wahrscheinlichkeit nach der Geburt eines Kindes unverheiratet sind. Die Gemeindegröße korreliert stark positiv mit nichtehelicher Mutterschaft. Der Vergleich der Modelle 1 und 3 zeigt, dass die festgestellten Ost-West-Differenzen genauso wie Veränderungen über die Jahre

12 Die abhängige Variable im Logitmodell ist der Odds. Der Odds gibt das Verhältnis der Wahrscheinlichkeit zur Gegenwahrscheinlichkeit, dass ein Ereignis eintritt, an. Die Koeffizienten im Logitmodell (Odds Ratios) benennen entsprechend den Einfluss der unabhängigen Variablen auf ein Chancenverhältnis. Positive Koeffizienten bedeuten einen positiven Zusammenhang, negative Koeffizienten einen negativen Zusammenhang zwischen unabhängiger Variable und der Wahrscheinlichkeit des Eintritts eines Ereignisses (siehe z.B. Andreß et al. 1997). 
weitgehend unabhängig vom Alter bei der Mutterschaft, der Nationalität, dem Wohnort und dem Bildungsniveau der Frau bestehen.

Der Bildungsabschluss ist eine aus theoretischer Sicht besonders relevante Determinante der Familienform. Man erkennt eine leicht U-förmige Beziehung zwischen dem formalen Bildungsniveau und dem Familienstand der Frauen. Auf der einen Seite verringert ein Realschulabschluss die Wahrscheinlichkeit ledig zu sein im Vergleich zur Referenzkategorie der Frauen, die ein Abitur besitzen. Auf der anderen Seite bleiben Frauen mit Hauptschulabschluss bzw. ohne Schulabschluss eher ledig als die Frauen der Referenzkategorie. Der Unterschied zwischen den Frauen mit Abitur und jenen mit Hauptschulabschluss bzw. ohne Schulabschluss ist jedoch nicht signifikant.

Um zu überprüfen, in welchem Ausmaß sich die sozialstrukturellen Determinanten lediger Mutterschaft in Ost- und Westdeutschland unterscheiden, haben wir in einem weiteren Schritt getrennte Analysen durchgeführt (Modelle 4a und 4b). Die Odds ledig zu sein sind in Ost- und Westdeutschland im Lauf der 1990er Jahre gestiegen. Auch die anderen Einflussfaktoren weisen in beiden Landesteilen in die gleiche Richtung. So ist die Größe des Wohnorts positiv und die nichtdeutsche Staatsbürgerschaft negativ mit der Wahrscheinlichkeit einer ledigen Mutterschaft verknüpft. In Ost- und Westdeutschland sind Mütter unter 21 Jahren sehr viel häufiger unverheiratet als ältere Mütter.

Ost-West-Unterschiede bestehen dagegen in der Bedeutung des Bildungsniveaus. In Westdeutschland zeigt sich - wie bereits in Modell 3 - eine bipolare Struktur des Bildungseffekts, d.h. sowohl eine niedrige als auch eine höhere allgemeine Bildung fördern ledige Mutterschaft. In Ostdeutschland ist dagegen der Bildungseffekt negativ. So erhöht sich der Odds im Vergleich zur oberen Bildungskategorie (Abitur) um annähernd 160 Prozent, wenn die Befragten über einen Hauptschul- oder keinen Abschluss verfügen und um fast 40 Prozent, wenn sie über einen Realschulabschluss oder einen Abschluss der Polytechnischen Oberschule verfügen. Frauen, die keinen Bildungsabschluss oder nur einen Hauptschulabschluss haben, sind demnach in Ostdeutschland mit Abstand am häufigsten nach der Geburt eines Kindes ledig. Der Bildungseffekt ist zugleich mit Vorsicht zu interpretieren, da in Ostdeutschland nur wenige Frauen über keinen Schulabschluss bzw. einen Hauptschulabschluss verfügen, die untere Bildungsgruppe also sozial selektiver als in Westdeutschland ist (siehe 
Tabelle 2). Dessen ungeachtet ist die Wahrscheinlichkeit ledig zu sein, für Mütter mit mittlerem Abschluss im Westen relativ niedrig und im Osten relativ hoch.

Tabelle 3: Determinanten lediger Mutterschaft bei Frauen mit einem Kind im Alter von 0-1 Jahren (logistische Regression, Odds Ratios). Abhängige Variable: ledig versus verheiratet (Referenzkategorie)

\begin{tabular}{|c|c|c|c|c|c|}
\hline & Modell 1 & Modell 2 & Modell 3 & $\begin{array}{l}\text { Modell 4a } \\
\text { - West - }\end{array}$ & $\begin{array}{c}\text { Modell 4b } \\
\text { - Ost - }\end{array}$ \\
\hline \multicolumn{6}{|l|}{ Region } \\
\hline Ostdeutschland & $6.34 * * *$ & $4.81 * * *$ & $5.54 * * *$ & & \\
\hline Westdeutschland & 1 & 1 & 1 & & \\
\hline \multicolumn{6}{|l|}{ Jahr } \\
\hline 1991 & 1 & 1 & 1 & 1 & 1 \\
\hline 1993 & $1.24 * * *$ & $1.34 * * *$ & $1.33 * * *$ & $1.23 * * *$ & $1.43 * * *$ \\
\hline 1996 & $1.60 * * *$ & $1.92 * * *$ & $1.96 * * *$ & $1.67 * * *$ & $2.74 * * *$ \\
\hline 1998 & $1.93 * * *$ & $2.41 * * *$ & $2.49 * * *$ & $2.14 * * *$ & $3.37 * * *$ \\
\hline 2000 & $2.45 * * *$ & $3.02 * * *$ & $3.15 * * *$ & $2.64 * * *$ & $4.54 * * *$ \\
\hline \multicolumn{6}{|l|}{ Anzahl der Kinder } \\
\hline Ein Kind & 1 & 1 & 1 & 1 & 1 \\
\hline Zwei oder mehr Kinder & $0.22 * * *$ & $0.29 * * *$ & $0.29 * * *$ & $0.30 * * *$ & $0.29 * * *$ \\
\hline \multicolumn{6}{|l|}{ Alter } \\
\hline $16-20$ & & $6.74 * * *$ & $6.47 * * *$ & $6.35 * * *$ & $7.42 * * *$ \\
\hline $21-25$ & & $2.13 * * *$ & $2.13 * * *$ & $2.21 * * *$ & $2.07 * * *$ \\
\hline $26-30$ & & 1 & 1 & 1 & 1 \\
\hline $31-45$ & & $0.82 * * *$ & $0.80 * * *$ & $0.85 * * *$ & $0.62 * * *$ \\
\hline \multicolumn{6}{|l|}{ Nationalität } \\
\hline Deutsch & & 1 & 1 & 1 & 1 \\
\hline Andere & & $0.28 * * *$ & $0.23 * * *$ & $0.23 * * *$ & $0.19 * * *$ \\
\hline \multicolumn{6}{|l|}{ Gemeindegröße } \\
\hline 0-20.000 Einwohner & & & 1 & 1 & 1 \\
\hline 20.000-100.000 E. & & & $1.29 * * *$ & $1.29 * * *$ & $1.29 * * *$ \\
\hline 100.000 u. m. E. & & & $2.39 * * *$ & $2.49 * * *$ & $1.95 * * *$ \\
\hline \multicolumn{6}{|l|}{ Bildungsabschluss } \\
\hline Hauptschule/kein Abschluss & & & 1.06 & 0.93 & $2.56 * * *$ \\
\hline Realschule/POS & & & $0.86 * *$ & $0.74 * * *$ & $1.38 * * *$ \\
\hline Abitur & & & 1 & 1 & 1 \\
\hline \multicolumn{6}{|l|}{ Modellgüte } \\
\hline Pseudo $r^{2}$ (nach McFadden) & 0.15 & 0.19 & 0.21 & 0.13 & 0.19 \\
\hline
\end{tabular}

Anmerkung: Die Stichproben umfassen Frauen, die zum Befragungszeitpunkt zwischen 16 und 45 Jahre alt sind und Kinder zwischen 0 und 1 Jahren alt haben, die im selben Haushalt leben. Für fehlende Angaben zum Bildungsniveau wurde kontrolliert.

$* * * \mathrm{p}<0.01 ; * * \mathrm{p}<0.05 ; * \mathrm{p}<0.10$

Quelle: Scientific-Use-File des Mikrozensus 1991, 1993, 1996, 1998, 2000 (eigene Berechnungen) 


\subsection{Sozialstrukturelle Determinanten der Familienform 1995-2000}

Unseren theoretischen Überlegungen zu Folge sollten sich alleinerziehende und kohabitierende Mütter in ihren sozialstrukturellen Hintergründen deutlich unterscheiden. Da mit dem Mikrozensus erst seit 1996 Personen, die in nichtehelichen Lebensgemeinschaften leben, identifizierbar sind, müssen wir uns auf die Auswertung der Jahre 1996 bis 2000 beschränken. In Tabelle 4 sind die Ergebnisse einer multinomialen Regression wiedergegeben, deren abhängige Variable die Familienform mit den drei Ausprägungen Ehe, nichteheliche Lebensgemeinschaft und alleinerziehend ist.

In Westdeutschland haben Frauen mit niedrigem Bildungsabschluss eine höhere Wahrscheinlichkeit als die anderen Bildungsgruppen, alleinerziehend zu sein. Zugleich ist für Frauen mit Abitur die Wahrscheinlichkeit, mit einem Kind in einer nichtehelichen Lebensgemeinschaft zu leben, größer als für die beiden anderen Bildungskategorien. Dieses Ergebnis bedeutet eine entscheidende Differenzierung der vorangegangenen Analysen auf der Grundlage der älteren Mikrozensen, in denen wir Alleinerziehende von Frauen in nichtehelichen Lebensgemeinschaften nicht unterscheiden konnten. Diese ergaben für Westdeutschland einen U-förmigen Zusammenhang zwischen dem Bildungsniveau und dem Familienstand der Frauen (Tabelle 3, Modell 4a). Wie nun deutlich wird, ist dieser auf die Aggregation von Frauen mit Kindern in nichtehelichen Lebensgemeinschaften und Alleinerziehenden zu einer einzigen Kategorie zurückzuführen. Getrennt analysiert, erweisen sich die sozialstrukturellen Grundlagen der beiden , neuen Familienformen' in Westdeutschland als sehr unterschiedlich. Es lässt sich eine polarisierte Struktur dieser Familienform im Hinblick auf die Bildungsressourcen der Frauen feststellen. Gilt dies auch für Ostdeutschland? 
Tabelle 4: Determinanten der Familienform von Frauen mit einem Kind im Alter von 0-1 Jahren (Multinomiale logistische Regression, Odds Ratios). Abhängige Variable: Alleinerziehend, NEL, verheiratet (Referenzkategorie)

\begin{tabular}{|c|c|c|c|c|}
\hline & \multicolumn{2}{|c|}{ Westdeutschland } & \multicolumn{2}{|c|}{ Ostdeutschland } \\
\hline & Alleinerziehend & NEL & Alleinerziehend & NEL \\
\hline \multicolumn{5}{|l|}{ Jahr } \\
\hline 1996 & 1 & 1 & 1 & 1 \\
\hline 1998 & 1.18 & $1.39 * * *$ & 1.22 & 1.25 \\
\hline 2000 & $1.41 * * *$ & $1.74 * * *$ & $1.60 * * *$ & $1.75 * * *$ \\
\hline \multicolumn{5}{|l|}{ Anzahl der Kinder } \\
\hline Ein Kind & 1 & 1 & 1 & 1 \\
\hline Zwei oder mehr Kinder & $0.30 * * *$ & $0.29 * * *$ & $0.36 * * *$ & $0.37 * * *$ \\
\hline \multicolumn{5}{|l|}{ Alter } \\
\hline $16-20$ & $8.53 * * *$ & $3.71 * * *$ & $13.21 * * *$ & $6.27 * * *$ \\
\hline $21-25$ & $2.41 * * *$ & $1.99 * * *$ & $2.35 * * *$ & $1.85 * * *$ \\
\hline $26-30$ & 1 & 1 & 1 & 1 \\
\hline $30-45$ & 0.85 & $0.81 * *$ & $0.63 * *$ & $0.50 * * *$ \\
\hline \multicolumn{5}{|l|}{ Nationalität } \\
\hline Deutsch & 1 & 1 & 1 & 1 \\
\hline Andere & $0.20 * * *$ & $0.30 * * *$ & $0.05 * * *$ & $0.14 * * *$ \\
\hline \multicolumn{5}{|l|}{ Gemeindegröße } \\
\hline 0-20.000 Einwohner & 1 & 1 & 1 & 1 \\
\hline $20.000-100.000$ E. & $1.60 * * *$ & 1.12 & $1.35 *$ & $1.40 * * *$ \\
\hline 100.000 u. m. E. & $3.31 * * *$ & $1.82 * * *$ & $2.56 * * *$ & $1.96 * * *$ \\
\hline \multicolumn{5}{|l|}{ Bildungsabschluss } \\
\hline Hauptschule/kein Abschluss & $1.54 * * *$ & $0.66 * * *$ & $6.11 * * *$ & $1.85 * *$ \\
\hline Realschule/POS & 0.95 & $0.63 * * *$ & $1.93 * * *$ & 1.13 \\
\hline Abitur & 1 & 1 & 1 & 1 \\
\hline \multicolumn{5}{|l|}{ Modellgüte } \\
\hline Pseudo $^{2}$ (nach McFadden) & 0.11 & & 0.13 & \\
\hline
\end{tabular}

Anmerkung: Die Stichproben umfassen Frauen, die zum Befragungszeitpunkt zwischen 16 und 45 Jahre alt sind und Kinder zwischen 0 und 1 Jahren alt haben, die im selben Haushalt leben. Für fehlende Angaben zum Bildungsniveau wurde kontrolliert.

$* * * \mathrm{p}<0.01 ; * * \mathrm{p}<0.05 ; * \mathrm{p}<0.10$

Quelle: Scientific Use File des Mikrozensus 1996, 1998, 2000 (eigene Berechnungen) 
Zunächst ist festzustellen, dass der Zusammenhang zwischen dem Bildungsniveau und alleinerziehender Mutterschaft auch in Ostdeutschland negativ ist. Er ist jedoch bei genauerem Hinsehen etwas anders strukturiert. In Westdeutschland sind es die Frauen mit Hauptschulabschluss (bzw. ohne Abschluss), die mit einer höheren Wahrscheinlichkeit alleinerziehend sind, während sich Frauen mit Realschulabschluss und Abitur nicht signifikant voneinander unterscheiden. In Ostdeutschland sind demgegenüber die Odds alleinerziehend $\mathrm{zu}$ sein für Frauen mit mittlerem Bildungsabschluss (POS bzw. Realschulabschluss) doppelt so hoch wie für Frauen mit Abitur. Mit Abstand die größte Wahrscheinlichkeit, allein mit einem 0- bis 1-jährigen Kind zu leben, weisen schließlich die Frauen mit Hauptschul- bzw. ohne Schulabschluss auf.

Anders als in Westdeutschland beeinflusst auch das formale Bildungsniveau die Wahrscheinlichkeit, in einer nichtehelichen Lebensgemeinschaft zu leben. Während in Westdeutschland eine geringe oder mittlere Bildung die entsprechenden Odds negativ beeinflusst, erhöht im Osten eine geringe allgemeine Schulbildung die Wahrscheinlichkeit, dass eine Frau mit einem Kind in einer nichtehelichen Lebensgemeinschaft lebt. Frauen mit mittleren Schulabschluss unterscheiden sich zugleich kaum von jenen mit Abitur. Zu berücksichtigen ist in diesem Zusammenhang wiederum, dass die untere Bildungsgruppe in Ostdeutschland sehr klein und nicht direkt mit den westdeutschen Hauptschulabsolventinnen zu vergleichen ist.

Die bisherigen Analysen bestätigen für Ost- und Westdeutschland die Annahme, dass Frauen, die über ein niedriges formales Bildungsniveau verfügen und früh Mutter werden, eine erhöhte Wahrscheinlichkeit aufweisen, nach der Geburt eines Kindes alleinerziehend zu sein (Hypothese 2a). In Westdeutschland haben alleinerziehende und kohabitierende Mütter erwartungsgemäß sozialstrukturell sehr unterschiedliche Charakteristika. So leben die höher gebildeten Frauen häufiger als die anderen Gruppen auch nach der Familiengründung in einer nichtehelichen Lebensgemeinschaft (Hypothese 1a). In Ostdeutschland ist dieser Zusammenhang jedoch nicht festzustellen. Für Frauen mit Abitur ist die Wahrscheinlichkeit, in einer nichtehelichen Lebensgemeinschaft zu leben, genauso groß wie für Frauen mit mittlerem Abschluss. Hinzu kommt, dass in Ostdeutschland Frauen mit geringen Bildungsressourcen am häufigsten in einer nichtehelichen Lebensgemeinschaft leben. 


\subsection{Die ökonomischen Grundlagen neuer Familienformen 2000}

Im Anschluss an die Analyse des formalen Bildungsniveaus untersuchen wir im Folgenden die Erwerbsbeteiligung und den überwiegenden Lebensunterhalt der Frauen in den Jahren nach der Geburt eines Kindes. Im Unterschied zur Frage der Determinanten einer ehelichen bzw. nichtehelichen Geburt adressieren wir nun die Frage nach dem Ausmaß der ökonomischen Unabhängigkeit von Frauen in der frühen Familienphase. Wir beschränken uns auf die Daten des Mikrozensus 2000 und beziehen alle Frauen in die Analyse ein, die zwischen 16 und 45 Jahre alt sind und ein Kind im Alter von bis zu 10 Jahren haben, das im selben Haushalt lebt. Die drei Familienformen werden nach dem Alter des jüngsten Kindes (0 bis 2 Jahre, 3 bis 6 Jahre und 7 bis 10 Jahre) differenziert. ${ }^{13}$ Auf diese Weise berücksichtigen wir, dass das Erwerbsverhalten von Müttern und insofern auch das Ausmaß der Abhängigkeit von Transferleistungen bzw. der Unterstützung durch den Partner mit dem Alter des Kindes variiert. Vor allem in Westdeutschland schränkt die schlechte Betreuungsinfrastruktur für unter 3-jährige Kinder die Chancen der Erwerbsarbeit von Müttern stark ein.

Tabelle 5 gibt zunächst den Erwerbsstatus von Müttern nach dem Alter ihres jüngsten Kindes und der Familienform wieder. ${ }^{14}$ In Westdeutschland sind Frauen in nichtehelichen Lebensgemeinschaften, aber auch Alleinerziehende erwartungsgemäß häufiger Vollzeit erwerbstätig als verheiratete Frauen. Die entsprechenden Differenzen kristallisieren sich umso deutlicher heraus, je älter das jüngste Kind ist. In Ostdeutschland ist das Ausmaß der Vollzeiterwerbstätigkeit von Müttern insgesamt größer. Zugleich bestehen nur geringe Unterschiede im Erwerbsverhalten der Frauen in den verschiedenen Familienformen. Im Unterschied $\mathrm{zu}$ Westdeutschland sind die Alleinerziehenden in der Tendenz seltener Vollzeit erwerbstätig als die verheirateten oder kohabitierenden Frauen. Die größten Ost-West-Unterschiede bestehen im Erwerbsverhalten verheirateter Frauen mit Kindern im Alter von 7 bis 10 Jahren. 60

13 Frauen, die im Jahr 2000 Kinder im Alter von 0 bis 10 Jahren hatten, haben diese zwischen 1989 und 2000 geboren. Die folgenden Analysen beruhen damit annäherungsweise auf der gleichen Untersuchungspopulation wie die bisherigen Analysen.

14 Den Erwerbsstatus haben wir aus den Angaben zum Erwerbsstatus (ef110), zur tatsächlichen Wochenarbeitszeit in der Berichtswoche (ef143), und Informationen darüber, ob die Befragte arbeitsuchend ist (ef208), generiert. Teilzeit erwerbstätig sind Personen, die in der Berichtswoche zwischen 1 bis unter 30 Stunden erwerbstätig sind. Vollzeit erwerbstätig sind Personen, die 30 und mehr Stunden pro Woche erwerbstätig sind. Nicht erwerbstätig sind Personen, die angeben, nicht erwerbstätig zu sein oder eine tatsächliche wöchentliche Arbeitszeit von 0 Stunden haben. 
Prozent der verheirateten Frauen in Ostdeutschland, aber nur 16 Prozent der Frauen dieser Kategorie in Westdeutschland sind Vollzeit erwerbstätig. Ein weiterer Ost-WestUnterschied besteht darin, dass die nichterwerbstätigen Frauen in den neuen Ländern vor allem, wenn das jüngste Kind mindestens drei Jahre alt ist - sehr viel häufiger arbeitssuchend sind als in den alten Ländern.

Tabelle 5: Erwerbsstatus von Frauen nach der Familienform im Jahr 2000 (Spaltenprozente)

\begin{tabular}{|c|c|c|c|c|c|c|}
\hline & \multicolumn{3}{|c|}{ Westdeutschland } & \multicolumn{3}{|c|}{ Ostdeutschland } \\
\hline & $\begin{array}{c}\text { Allein- } \\
\text { erziehend }\end{array}$ & NEL & $\begin{array}{c}\text { Verhei- } \\
\text { ratet }\end{array}$ & $\begin{array}{c}\text { Allein- } \\
\text { erziehend }\end{array}$ & NEL & $\begin{array}{c}\text { Verhei- } \\
\text { ratet }\end{array}$ \\
\hline \multicolumn{7}{|l|}{ Alle } \\
\hline Vollzeit erwerbstätig & $26 \%$ & $20 \%$ & $12 \%$ & $32 \%$ & $38 \%$ & $45 \%$ \\
\hline Teilzeit erwerbstätig & $21 \%$ & $28 \%$ & $32 \%$ & $7 \%$ & $9 \%$ & $13 \%$ \\
\hline \multicolumn{7}{|l|}{ Nicht erwerbstätig } \\
\hline -- Arbeitssuchend & $13 \%$ & $7 \%$ & $4 \%$ & $27 \%$ & $19 \%$ & $19 \%$ \\
\hline -- Andere & $41 \%$ & $46 \%$ & $51 \%$ & $34 \%$ & $34 \%$ & $23 \%$ \\
\hline Insgesamt & $100 \%$ & $100 \%$ & $100 \%$ & $100 \%$ & $100 \%$ & $100 \%$ \\
\hline \multicolumn{7}{|l|}{ Jüngstes Kind 0-2 } \\
\hline Vollzeit erwerbstätig & $11 \%$ & $9 \%$ & $8 \%$ & $13 \%$ & $17 \%$ & $20 \%$ \\
\hline Teilzeit erwerbstätig & $11 \%$ & $20 \%$ & $15 \%$ & $3 \%$ & $8 \%$ & $10 \%$ \\
\hline \multicolumn{7}{|l|}{ Nicht erwerbstätig } \\
\hline -- Arbeitssuchend & $7 \%$ & $5 \%$ & $2 \%$ & $16 \%$ & $12 \%$ & $10 \%$ \\
\hline -- Andere & $71 \%$ & $66 \%$ & $76 \%$ & $68 \%$ & $63 \%$ & $61 \%$ \\
\hline Insgesamt & $100 \%$ & $100 \%$ & $100 \%$ & $100 \%$ & $100 \%$ & $100 \%$ \\
\hline \multicolumn{7}{|l|}{\begin{tabular}{|l} 
Jüngstes Kind 3-6 \\
\end{tabular}} \\
\hline Vollzeit erwerbstätig & $29 \%$ & $27 \%$ & $13 \%$ & $39 \%$ & $52 \%$ & $47 \%$ \\
\hline Teilzeit erwerbstätig & $24 \%$ & $35 \%$ & $36 \%$ & $9 \%$ & $12 \%$ & $14 \%$ \\
\hline \multicolumn{7}{|l|}{ Nicht erwerbstätig } \\
\hline -- Arbeitssuchend & $17 \%$ & $9 \%$ & $5 \%$ & $36 \%$ & $29 \%$ & $25 \%$ \\
\hline -- Andere & $30 \%$ & $28 \%$ & $46 \%$ & $17 \%$ & $7 \%$ & $14 \%$ \\
\hline Insgesamt & $100 \%$ & $100 \%$ & $100 \%$ & $100 \%$ & $100 \%$ & $100 \%$ \\
\hline \multicolumn{7}{|l|}{\begin{tabular}{|l|} 
Jüngstes Kind 7-10 \\
\end{tabular}} \\
\hline Vollzeit erwerbstätig & $41 \%$ & $43 \%$ & $16 \%$ & $51 \%$ & $65 \%$ & $60 \%$ \\
\hline Teilzeit erwerbstätig & $29 \%$ & $38 \%$ & $45 \%$ & $10 \%$ & $9 \%$ & $14 \%$ \\
\hline \multicolumn{7}{|l|}{ Nicht erwerbstätig } \\
\hline -- Arbeitssuchend & $14 \%$ & $7 \%$ & $5 \%$ & $33 \%$ & $22 \%$ & $19 \%$ \\
\hline -- Andere & $16 \%$ & $11 \%$ & $33 \%$ & $6 \%$ & $4 \%$ & $7 \%$ \\
\hline Insgesamt & $100 \%$ & $100 \%$ & $100 \%$ & $100 \%$ & $100 \%$ & $100 \%$ \\
\hline
\end{tabular}

Anmerkung: Die Stichproben umfassen Frauen, die zum Befragungszeitpunkt zwischen 16 und 45 Jahre alt sind und Kinder im Alter von 0 bis 10 Jahren haben, die im selben Haushalt leben.

Quelle: Scientific-Use-File des Mikrozensus 2000 (eigene Berechnungen) 
Um die Frage nach der ökonomischen Unabhängigkeit von Frauen mit Kindern genauer beantworten zu können, ziehen wir zusätzlich die im Mikrozensus enthaltenen Informationen über die Quelle des überwiegenden Lebensunterhalts heran. Die entsprechende Variable erlaubt eine Differenzierung zwischen der eigenen Erwerbstätigkeit, dem Einkommen des Partners bzw. der Angehörigen und sozialstaatlichen Transferzahlungen. Entsprechend unterscheiden wir im Folgenden drei Statusgruppen: erstens Frauen, die ihren überwiegenden Lebensunterhalt durch eigene Erwerbstätigkeit oder eigenes Vermögen bestreiten, zweitens Frauen, die überwiegend vom Unterhalt durch den Partner oder Angehörige leben und drittens Frauen, die ihren überwiegenden Lebensunterhalt durch staatliche Transferzahlungen bestreiten. Die letztere Kategorie differenzieren wir zusätzlich danach, ob der überwiegende Lebensunterhalt aus Arbeitslosengeld/-hilfe oder Transferzahlungen wie der Sozialhilfe oder dem Bafög besteht (Tabelle 6). ${ }^{15}$ Die Unterscheidung von drei hauptsächlichen Quellen des Lebensunterhalts entspricht der Differenzierung von Markt, Staat und Familie, wie sie als Basisinstitutionen sozialer Sicherung in der vergleichenden Wohlfahrtsstaatsforschung diskutiert werden (siehe Abschnitt 2 bzw. Abbildung 2). Die drei Familienformen werden erneut nach dem Alter des jüngsten Kindes differenziert.

In Westdeutschland verfügen den Angaben über die Herkunft der Mittel für den eigenen Lebensunterhalt $\mathrm{zu}$ Folge Mütter, die in einer nichtehelichen Lebensgemeinschaft leben, über das relativ größte Ausmaß ökonomischer Unabhängigkeit. Wenn das jüngste Kind 3 bis 6 bzw. 7 bis 10 Jahre alt ist, bestreiten zwei Drittel (63\%) bzw. vier Fünftel (79\%) dieser Frauen ihren Lebensunterhalt überwiegend durch eigene Erwerbstätigkeit. Der staatliche Transferbezug hat für Frauen in nichtehelichen Lebensgemeinschaften mit 17 bzw. 13 Prozent nur eine geringe Bedeutung. Dagegen bestreitet knapp die Hälfte der Alleinerziehenden ihren

15 Es handelt sich um die Frage „Woraus beziehen Sie überwiegend die Mittel für Ihren Lebensunterhalt?“ (Variable ef122). Insbesondere für Personen mit verschiedenen Einkommensquellen hängt die Beantwortung der Frage zum überwiegenden Lebensunterhalt von der individuellen Einschätzung ihrer Einkommensquellen ab. Verheiratete Personen, die nur ein geringes eigenes Einkommen haben, werden durch den Interviewer angewiesen, den Unterhalt durch den Partner anzugeben. In Westdeutschland geben jedoch fast 30 Prozent der nicht bzw. marginal erwerbstätigen verheirateten Frauen an, dass sie ihren Lebensunterhalt durch eigene Erwerbstätigkeit (oder eigenes Vermögen) bestreiten. Es liegt nahe zu vermuten, dass in vielen Fällen Zuordnungsprobleme vorliegen und entsprechend in Tabelle 6 das Ausmaß der ökonomischen Unabhängigkeit der Befragten überschätzt wird. Der Vergleich der Tabellen 5 und 6 lässt darauf schließen, dass die meisten Teilzeit erwerbstätigen Frauen die eigene Erwerbsarbeit als überwiegende Quelle des eigenen Unterhalts angegeben haben. 
Lebensunterhalt durch Transfereinkommen, darunter hauptsächlich Sozialhilfe. Insgesamt 62 Prozent dieser Frauen leben von Transferzahlungen, wenn das jüngste Kind zwischen 0 und 2 Jahren alt ist. Dieser Anteil sinkt, wenn das Kind zwischen 3 und 6 Jahren ist, es bleiben aber 44 Prozent überwiegend auf staatliche Leistungen angewiesen.

In Ostdeutschland spielt der Transferbezug für Frauen mit Kindern im Alter zwischen 0 und 2 Jahren in allen Familienformen eine weitaus größere Rolle als in Westdeutschland. Für 82 Prozent aller Alleinerziehenden und 65 Prozent aller Mütter in nichtehelichen Lebensgemeinschaften, aber auch für 50 Prozent der Mütter in ehelichen Lebensgemeinschaften sind Transferleistungen die überwiegende Quelle des Lebensunterhalts. Im Unterschied zu den Frauen in Westdeutschland beziehen sie sehr häufig , andere' Transferzahlungen jenseits der Sozialhilfe. Diese Transferzahlungen werden im Mikrozensus nicht genauer aufgeschlüsselt, es dürfte sich aber überwiegend um Leistungen nach dem Erziehungsgeldgesetz handeln. Dem Partner bzw. anderen Familienangehörigen kommt in Ostdeutschland als Unterhaltsquelle durchweg eine geringe Bedeutung zu. Dies gilt überraschenderweise auch für verheiratete Frauen, die mit 15 Prozent zwar häufiger als kohabitierende Frauen (6\%), aber deutlich seltener als verheiratete Frauen in Westdeutschland (58\%) hauptsächlich vom Partnereinkommen leben. Mit steigendem Alter des jüngsten Kindes nimmt die Bedeutung des Transferbezugs in allen Familienformen ab. Wenn das Kind 3 bis 6 Jahre alt ist, spielt der Sozialhilfebezug mit 12 Prozent als Unterhaltsquelle für alleinerziehende Frauen in Ostdeutschland nur noch eine untergeordnete Rolle - im Unterschied $\mathrm{zu}$ den Alleinerziehenden in Westdeutschland, von denen immer noch 30 Prozent von Sozialhilfe leben. In Ostdeutschland erhält dagegen die Arbeitslosenunterstützung eine vergleichsweise große Bedeutung. 
Tabelle 6: $\quad$ Überwiegender Lebensunterhalt von Frauen nach der Familienform im Jahr 2000 (Spaltenprozente)

\begin{tabular}{|c|c|c|c|c|c|c|}
\hline & \multicolumn{3}{|c|}{ Westdeutschland } & \multicolumn{3}{|c|}{ Ostdeutschland } \\
\hline & $\begin{array}{c}\text { Allein- } \\
\text { erziehend }\end{array}$ & NEL & $\begin{array}{c}\text { Verhei- } \\
\text { ratet }\end{array}$ & $\begin{array}{c}\text { Allein- } \\
\text { erziehend }\end{array}$ & NEL & $\begin{array}{l}\text { Verhei- } \\
\text { ratet }\end{array}$ \\
\hline \multicolumn{7}{|l|}{ Alle } \\
\hline Erwerbstätigkeit/ Vermögen & $47 \%$ & $56 \%$ & $41 \%$ & $37 \%$ & $49 \%$ & $60 \%$ \\
\hline Partner/ Angehörige & $7 \%$ & $26 \%$ & $54 \%$ & $1 \%$ & $4 \%$ & $9 \%$ \\
\hline \multicolumn{7}{|l|}{ Transferzahlungen } \\
\hline -- Arbeitslosengeld/-hilfe & $7 \%$ & $4 \%$ & $2 \%$ & $22 \%$ & $15 \%$ & $15 \%$ \\
\hline -- Sozialhilfe & $33 \%$ & $7 \%$ & $2 \%$ & $21 \%$ & $5 \%$ & $3 \%$ \\
\hline -- Andere & $6 \%$ & $7 \%$ & $1 \%$ & $19 \%$ & $28 \%$ & $13 \%$ \\
\hline Insgesamt & $100 \%$ & $100 \%$ & $100 \%$ & $100 \%$ & $100 \%$ & $100 \%$ \\
\hline \multicolumn{7}{|l|}{\begin{tabular}{|l|} 
Jüngstes Kind 0-2 \\
\end{tabular}} \\
\hline Erwerbstätigkeit/ Vermögen & $27 \%$ & $45 \%$ & $37 \%$ & $16 \%$ & $29 \%$ & $35 \%$ \\
\hline Partner/ Angehörige & $11 \%$ & $34 \%$ & $58 \%$ & $2 \%$ & $6 \%$ & $15 \%$ \\
\hline \multicolumn{7}{|l|}{ Transferzahlungen } \\
\hline -- Arbeitslosengeld/-hilfe & $3 \%$ & $2 \%$ & $1 \%$ & $5 \%$ & $4 \%$ & $4 \%$ \\
\hline -- Sozialhilfe & $48 \%$ & $8 \%$ & $3 \%$ & $40 \%$ & $8 \%$ & $6 \%$ \\
\hline -- Andere & $11 \%$ & $10 \%$ & $2 \%$ & $37 \%$ & $53 \%$ & $40 \%$ \\
\hline Insgesamt & $100 \%$ & $100 \%$ & $100 \%$ & $100 \%$ & $100 \%$ & $100 \%$ \\
\hline \multicolumn{7}{|l|}{ Jüngstes Kind 3-6 } \\
\hline Erwerbstätigkeit/Vermögen & $50 \%$ & $63 \%$ & $40 \%$ & $45 \%$ & $61 \%$ & $62 \%$ \\
\hline Partner/ Angehörige & $6 \%$ & $20 \%$ & $55 \%$ & $1 \%$ & $4 \%$ & $10 \%$ \\
\hline \multicolumn{7}{|l|}{ Transferzahlungen } \\
\hline -- Arbeitslosengeld/-hilfe & $10 \%$ & $6 \%$ & $2 \%$ & $33 \%$ & $26 \%$ & $19 \%$ \\
\hline -- Sozialhilfe & $30 \%$ & $7 \%$ & $2 \%$ & $12 \%$ & $1 \%$ & $2 \%$ \\
\hline -- Andere & $4 \%$ & $4 \%$ & $0 \%$ & $9 \%$ & $8 \%$ & $6 \%$ \\
\hline Insgesamt & $100 \%$ & $100 \%$ & $100 \%$ & $100 \%$ & $100 \%$ & $100 \%$ \\
\hline \multicolumn{7}{|l|}{ Jüngstes Kind 7-10 } \\
\hline Erwerbstätigkeit/Vermögen & $69 \%$ & $79 \%$ & $48 \%$ & $58 \%$ & $76 \%$ & $74 \%$ \\
\hline Partner/ Angehörige & $2 \%$ & $9 \%$ & $49 \%$ & $0 \%$ & $0 \%$ & $6 \%$ \\
\hline \multicolumn{7}{|l|}{ Transferzahlungen } \\
\hline -- Arbeitslosengeld/hilfe & $8 \%$ & $7 \%$ & $2 \%$ & $33 \%$ & $23 \%$ & $18 \%$ \\
\hline -- Sozialhilfe & $17 \%$ & $3 \%$ & $1 \%$ & $5 \%$ & $0 \%$ & $1 \%$ \\
\hline -- Andere & $5 \%$ & $3 \%$ & $0 \%$ & $3 \%$ & $1 \%$ & $2 \%$ \\
\hline Insgesamt & $100 \%$ & $100 \%$ & $100 \%$ & $100 \%$ & $100 \%$ & $100 \%$ \\
\hline
\end{tabular}

Anmerkung: Die Stichproben umfassen Frauen, die zum Befragungszeitpunkt zwischen 16 und 45 Jahre alt sind und Kinder im Alter von 0 bis 10 Jahren haben, die im selben Haushalt leben.

Quelle: Scientific-Use-File des Mikrozensus 2000 (eigene Berechnungen)

Insgesamt ist festzustellen, dass in Westdeutschland Frauen, die nach der Geburt eines Kindes in einer nichtehelichen Lebensgemeinschaft leben, in relativ hohem Ausmaß erwerbstätig und, gemessen am überwiegenden Lebensunterhalt, ökonomisch eigenständig bleiben. Unsere Erwartung, dass diese Frauen überwiegend das Doppelversorger-Modell realisieren, wird damit gestützt (Hypothese 1b). Dagegen bestreitet die Mehrheit der verheirateten Mütter den eigenen Lebensunterhalt 
überwiegend durch das Einkommen des Partners. Die Unterschiede zwischen beiden Gruppen im Ausmaß der Vollzeiterwerbstätigkeit und der primären Einkommensquelle sind am deutlichsten, wenn das jüngste Kind älter als sechs Jahre ist. In Ostdeutschland ist der Grad der Erwerbstätigkeit und der ökonomischen Eigenständigkeit von kohabitierenden Frauen noch höher als in Westdeutschland. Der eigentlich überraschende Befund sind jedoch die geringen Differenzen in Bezug auf das Erwerbsverhalten und die Abhängigkeit vom Partnereinkommen zwischen verheirateten und kohabitierenden Frauen in Ostdeutschland. 55 Prozent der Frauen in Westdeutschland, aber nur 10 Prozent der verheirateten Frauen in Ostdeutschland mit einem Kind im Alter zwischen 3 und 6 Jahren geben an, überwiegend vom Einkommen des Partners zu leben. Demnach herrscht das Doppel-Ernährer-Modell in den neuen Ländern relativ unabhängig von der Familienform vor. Die geringen Anteile der verheirateten Frauen, die vom Einkommen des Ehepartners leben, erfordern auch eine Spezifizierung von Hypothese $1 b$ im Hinblick auf die ostdeutsche Situation. Die sozialpolitischen Rahmenbedingungen in Deutschland binden zwar das MaleBreadwinner-Modell eng an die Lebensform der Ehe. Dennoch erfolgt die Entscheidung für die Ehe in Ostdeutschland weitgehend unabhängig von diesem Modell.

\section{Schlussfolgerungen}

Nichteheliche Mutterschaft wird im sozialpolitischen Diskurs sehr unterschiedlich interpretiert. Der vor allem in den USA und Großbritannien verbreiteten ,WelfareMother-Perspektive' steht die These des Funktionsverlustes der Ehe in Folge der zunehmenden ökonomischen Unabhängigkeit von Frauen gegenüber. Wir haben argumentiert, dass der Status ,nicht verheiratet mit Kind" sowohl im Hinblick auf die familiale Lebensform als auch den sozialstrukturellen Hintergrund genauer betrachtet werden sollte. Nichteheliche Mutterschaft wird überdies in differenzierter Weise staatlich reguliert. Unter den sozialpolitischen Rahmenbedingungen in Deutschland steht die Ehe als Lebensform unter dem besonderen Schutz des Staates, weshalb finanzielle Leistungen zugunsten ehelicher Lebensgemeinschaften nicht auf eheähnliche Lebensgemeinschaften ausgedehnt werden. Außerdem werden Alleinerziehende sozialpolitisch anders behandelt als Mütter, die mit einem Partner zusammen leben. 
Die hervorgehobene Stellung der Ehe in der Sozial- und Familienpolitik dürfte ein wesentlicher Grund für die im Unterschied zu vielen europäischen Ländern nach wie vor zentrale Rolle der Ehe als familialer Lebensform in Deutschland sein. Der Anstieg der nichtehelichen Geburten vor allem in den neuen Bundesländern in den 1990er Jahren verweist jedoch auf einen rasanten Prozess des Wandels der Familienformen. Wir haben in diesem Beitrag nichteheliche Mutterschaft in Ost- und Westdeutschland genauer im Hinblick auf die Differenzierung neuer Familienformen und die damit einhergehenden sozioökonomischen Ungleichheiten untersucht. Mit Hilfe der Daten der Scientific-Use-Files verschiedener Mikrozensen haben wir die sozialstrukturellen Hintergründe der neuen Familienformen und anschließend die ökonomische Position der Frau in der Familie betrachtet.

Die Analysen haben gezeigt, dass ein geringer Bildungsabschluss in Ost- und Westdeutschland die Wahrscheinlichkeit erhöht, dass Frauen nach der Geburt eines Kindes alleinerziehend sind. Uneinheitlich sind dagegen die Bestimmungsfaktoren einer nichtehelichen Lebensgemeinschaft. So leben im Westen Frauen mit Abitur mit einer höheren Wahrscheinlichkeit in einer nichtehelichen Lebensgemeinschaft als Frauen mit einem mittleren oder niedrigen Bildungsabschluss. Im Osten gilt dieser Zusammenhang jedoch nicht. Frauen mit Abitur kohabitieren nach der Geburt eines Kindes nicht häufiger als Frauen mit mittlerem Schulabschluss. Am größten ist schließlich die Wahrscheinlichkeit für Mütter mit niedrigem oder ohne Schulabschluss, in einer nichtehelichen Lebensgemeinschaft $\mathrm{zu}$ leben. Dieses Ergebnis widerspricht der Erwartung, dass eine höhere formale Bildung - als Indikator für eine verstärkte Orientierung auf ökonomische Unabhängigkeit - die Entscheidung für eine nichteheliche Lebensgemeinschaft als Familienform positiv beeinflusst. Jedoch ist die untere Bildungskategorie in Ostdeutschland schwach besetzt, und der Mikrozensus erlaubt es nicht, die zu DDR-Zeiten erworbenen Abschlüsse der POS nach der Abgangsklasse (8. oder 10. Klasse) zu differenzieren. Aus diesem Grund haben die unteren und mittleren Bildungskategorien in Ost und West nicht die gleiche Bedeutung. Dessen ungeachtet leben in Ostdeutschland Frauen mit mittlerem Abschluss - die bis zu drei Viertel der betrachteten Mütter repräsentieren - in gleichem Ausmaß in einer nichtehelichen Lebensgemeinschaft wie Frauen mit Abitur. Dieses Ergebnis verweist darauf, dass die nichteheliche Lebensgemeinschaft im Unterschied zu Westdeutschland als Familienform nicht auf die oberen Bildungsschichten konzentriert, sondern in der ,breiten Mitte' der Frauen verankert ist. 
Weiterhin bestehen Differenzen zwischen den verschiedenen Familienformen in Bezug auf das Erwerbsverhalten der Frauen und die Quellen ihres überwiegenden Lebensunterhalts. Kohabitierende Mütter und Alleinerziehende unterscheiden sich im Ausmaß, in dem sie erwerbstätig und von wohlfahrtsstaatlichen Transfereinkommen abhängig sind. In Westdeutschland ist die ökonomische Eigenständigkeit von Müttern ein typisches Merkmal der nichtehelichen Lebensgemeinschaft. Kohabitierende Frauen bleiben nach der Familiengründung in weitaus höherem $\mathrm{Maß}$ erwerbstätig als verheiratete Frauen, und sie hängen, eng damit verbunden, seltener vom Einkommen des Partners ab. Je älter das jüngste Kind ist, umso deutlicher kristallisiert sich dieser Zusammenhang heraus. Dieses Ergebnis entspricht der Annahme, dass die nichteheliche Lebensgemeinschaft als Familienform zugleich auf das Doppel-Ernährer-Modell verweist. Für Ostdeutschland haben wir zwei spezifische Merkmale der ökonomischen Basis der Familie identifizieren können. Zum einen spielen sozialstaatliche Transferleistungen in allen Familienformen eine größere Rolle als in Westdeutschland. Für Frauen mit Kindern über zwei Jahren hat der Bezug von Arbeitslosengeld/-hilfe eine große Bedeutung als Unterhaltsquelle. Dies betrifft nicht nur die Frauen in den neuen Familienformen, sondern in erheblichem Maß auch verheiratete Mütter. Zum anderen ist in den neuen Ländern der Anteil der verheirateten Frauen, die vom Einkommen des Partners leben, sehr niedrig. In Ostdeutschland ist demnach die Eheschließung kaum mit einer Entscheidung für das Male-Breadwinner-Modell der Familie verknüpft. Vielmehr haben sich die Ehe auf der einen Seite und das Ernährermodell auf der anderen Seite weitgehend voneinander entkoppelt.

Im Ost-West-Vergleich gibt es nur wenige Unterschiede in der ökonomischen Position von Müttern, die in einer nichtehelichen Lebensgemeinschaft leben. Verheiratete Mütter folgen dagegen in Ost- und Westdeutschland sehr unterschiedlichen Familienmodellen. Welche Gründe für diese Differenz verantwortlich sind, ist auf der Basis unserer empirischen Analysen nicht abschließend zu entscheiden. Plausibel sind u.E. zwei unterschiedliche Gründe. Eine Möglichkeit besteht darin, dass die hohe Erwerbsbeteiligung verheirateter Frauen in Ostdeutschland weniger auf das Streben nach ökonomischer Unabhängigkeit als auf eine schwächere Arbeitsmarktposition von Männern in Ostdeutschland verweist. Demnach wäre es vorrangig die Notwendigkeit eines zweiten Erwerbseinkommens in der Familie, das die höhere Erwerbsbeteiligung der verheirateten Frauen bedingt. Ein anderes Argument lautet, dass die ökonomische Eigenständigkeit der Frau ein notwendiges, aber kein hinreichendes Kriterium für die 
Entscheidung gegen die Ehe als Familienform darstellt. Zwar entfallen, wie wir argumentiert haben, durch die ökonomische Unabhängigkeit beider Partner zentrale finanzielle und sozialpolitische Vorteile der Ehe. Dessen ungeachtet bleiben jedoch weitere Gründe kultureller und rechtlicher Art für eine Eheschließung bestehen, welche die vergleichsweise hohen Anteile verheirateter und ökonomisch eigenständiger Frauen in Ostdeutschland erklären könnten.

Unabhängig von der Frage, welche Gründe für eine Eheschließung die ausschlaggebenden sind, lautet ein zentrales Ergebnis unseres Beitrags, dass der Wandel der Familie in den 1990er Jahren nicht nur in einer Zunahme nichtehelicher Geburten und neuer Familienformen besteht. Ein wichtiger Aspekt des Familienwandels ist in Westdeutschland auch die sozialstrukturelle Differenzierung nichtehelicher Mutterschaft mit den beiden Polen der Alleinerziehenden und der nichtehelichen Lebensgemeinschaften. In Ostdeutschland ist dies weniger der Fall. Dort vollzieht sich der familiale Wandel nicht zuletzt in Form einer durchgreifenden Modernisierung der ökonomischen Position von Frauen in der Familie, die nicht auf die unverheiratet mit einem Partner zusammenlebenden Mütter beschränkt ist. Während also nichteheliche Lebensgemeinschaften in Ost- und Westdeutschland gleichermaßen ein gewandeltes familiales Arbeitsteilungsmuster mit einer erhöhten ökonomischen Unabhängigkeit der Frauen aufweisen, hat sich in Ostdeutschland das Modell der ökonomischen Unabhängigkeit der Frau auch in der ehelichen Familie etabliert. Dieser Wandel in der Ehe bedingt, dass in Ostdeutschland den verschiedenen Familienformen kaum noch eine typische ökonomische Basis bzw. ein Versorger-Modell entspricht. Da die Durchsetzung eines Doppel-Ernährer-Modells die Organisationsform von Familie in ihrem Kern berührt, sollte die Frage der Erwerbsarbeitsteilung und der ökonomischen Position von Frauen eine zentrale Rolle in der Diskussion um den Wandel der Lebensund Familienformen spielen. 


\section{Literatur}

Andreß, Hans-Jürgen, Jacques A. Hagenaars und Steffen Kühnel, 1997: Analyse von Tabellen und kategorialen Daten. Log-lineare Modelle, latente Klassenanalyse, logistische Regression und GSK-Ansatz. Berlin, Heidelberg: Springer Verlag.

Becker, Gary S., 1993: A Treatise on the Family. Cambridge: Harvard University Press.

Blossfeld, Hans-Peter und Sonja Drobnič, 2001: Theoretical perspectives on couples' careers. S. 16-50 in: Dies. (Hg.), Careers of Couples in Contemporary Societies: From Male Breadwinner to Dual Earner Families. Oxford: Oxford University Press.

Brüderl, Josef und Thomas Klein, 2003: Die Pluralisierung partnerschaftlicher Lebensformen in Westdeutschland, 1960-2000. S. 189-217 in: Walter Bien und Jan H. Marbach (Hg.): Partnerschaft und Familiengründung. Ergebnisse der dritten Welle des Familien-Survey. Opladen: Leske und Budrich.

Büchel, Felix und Katharina C. Spieß, 2002: Formen der Kinderbetreuung und Arbeitsmarktverhalten von Müttern in West- und Ostdeutschland. Schriftenreihe des Bundesministeriums für Familie, Senioren, Frauen und Jugend Bd. 220. Stuttgart: Kohlhammer.

Cherlin, Andrew J., 2000: New developments in the study of nonmarital childbearing. S. 390402 in: Lawrence Wu und Barbara Wolfe (Hg.), Out of Wedlock: Causes and Consequences of Nonmarital Fertility. New York: Russell Sage.

Council of Europe, 2002: Recent Demographic Developments in Europe. Strasbourg: Council of Europe.

Daly, Mary, 2000: The Gender Division of Welfare: The Impact of the British and German Welfare State. Cambridge: Cambridge University Press.

Deutsches Jugendinstitut, 1993: Tageseinrichtungen für Kinder. Informationen, Erfahrungen, Analysen. München: DJI.

Deutsches Jugendinstitut, 2002: Zahlenspiegel: Daten zu Tageseinrichtungen für Kinder. München: DJI. 
Diewald, Martin und Sigrid Wehner, 1996: Verbreitung und Wechsel von Lebensformen im jüngeren Erwachsenenalter - Der Zeitraum von 1984 bis 1993. S. 125-146 in: Wolfgang Zapf, Jürgen Schupp und Roland Habich (Hg.), Lebenslagen im Wandel: Sozialberichterstattung im Längsschnitt. Frankfurt: Campus.

Dingeldey, Irene, 2001: European tax systems and their impact on family employment patterns. Journal of Social Policy 4: 563-672.

Dingeldey, Irene, 2002: Das deutsche System der Ehegattenbesteuerung im europäischen Vergleich. WSI-Mitteilungen 3: 154-160.

Dorbritz, Jürgen, 2000: Europäische Fertilitätsmuster. Zeitschrift für Bevölkerungswissenschaft 25: 235-266.

Esping-Andersen, Gøsta, 1990: The Three Worlds of Welfare Capitalism. Cambridge: Polity Press.

Esping-Andersen, Gøsta, 1999: Social Foundations of Postindustrial Economies. New York: Oxford University Press.

Fein, David J., 2001: Will welfare reform influence marriage and fertility? Early evidence from the ABC demonstration. Evaluation and Program Planning 24: 427-444.

Friedman, Debra, Michael Hechter und Satoshi Kanazawa, 1994: A theory of the value of children. Demography 31: 375-104.

Garfinkel, Irene, Huang Chien-Chung, Sara S. McLanahan und Daniel S. Gaylin, 2003: The roles of child support enforcement and welfare in non-marital childbearing. Journal of Population Economics 16: 55-70.

Gauthier, Anne H., 1996: The State and the Family: A Comparative Analysis of Family Policies in Industrialized Countries. Oxford: Oxford University Press.

Gerhard, Ute, 2003: Geschlecht: Frauen im Wohlfahrtsstaat. S. 267-285 in: Lessenich, Stephan (Hg.), Wohlfahrtsstaatliche Grundbegriffe. Frankfurt/M.: Campus.

Gornick, Janet C., Marcia K. Meyers und Kathrin E. Ross, 1998: Public policies and the employment of mothers: a cross national study. Social Science Quarterly 79: 35-54. 
Gysi, Jutta und Wulfram Speigner, 1983: Changes in the Life Patterns of Families in the German Democratic Republic. Berlin (Ost): Institut für Soziologie und Sozialpolitik an der Akademie der Wissenschaft der Deutschen Demokratischen Republik.

Hank, Karsten, Michaela Kreyenfeld und C. Katharina Spieß, 2004: Kinderbetreuung und Fertilität in Deutschland. Zeitschrift für Soziologie 34: 228-244.

Heidenreich, Hans-Joachim und Michaela Nöthen, 2002: Der Wandel der Lebensformen im Spiegel des Mikrozensus. Wirtschaft und Statistik, Heft 1: 26-38.

Höhn, Charlotte, 1992: Population relevant policies before and after unification in Germany. S. 4-23 in: Krishnan, P. et al. (Hg.): Readings in Population Research. B.R. Publishing, Dehli.

Höhn, Charlotte, und Jürgen Dorbritz, 1995: Zwischen Individualisierung und Institutionalisierung Familiendemographische Trends im vereinten Deutschland. S. 149-174 in: Nauck, Bernhard und Corinna Onnen-Isemann (Hg.), Familie im Brennpunkt von Wissenschaft und Forschung. Neuwied: Luchterhand.

Huinink, Johannes, Karl Ulrich Mayer und Heike Trappe, 1995: Staatliche Lenkung und individuelle Karrierechancen: Bildungs- und Berufsverläufe. S. 89-143 in: Johannes Huinink, Karl Ulrich Mayer u. a. (Hg.), Kollektiv und Eigensinn: Lebensverläufe in der DDR und danach, Berlin: Akademie Verlag.

Huinink, Johannes, 1999: Die Entscheidung zur Nichtehelichen Lebensgemeinschaft als Lebensform - Ein Vergleich zwischen Ost- und Westdeutschland. S. 113-138 in Thomas Klein und Wolfgang Lauterbach (Hg.), Nichteheliche Lebensgemeinschaften. Opladen: Leske und Budrich.

Huinink, Johannes und Dirk Konietzka, 2003: Lebensformen und Familiengründung Nichteheliche Elternschaft in West- und Ostdeutschland in den 1990er Jahren. S. 65-93 in: Walter Bien und Jan H. Marbach (Hg.): Partnerschaft und Familiengründung. Ergebnisse der dritten Welle des Familien-Survey. Opladen: Leske und Budrich.

Huinink, Johannes und Michael Wagner, 1998: Individualisierung und die Pluralisierung von Lebensformen. S. 85-106 in: Jürgen Friedrichs (Hg), Die Individualisierungs-These. Opladen: Leske + Budrich.

Kaufmann, Franz-Xaver, Anton Kuijsten, Hans-Joachim Schulze und Klaus Peter Strohmeier, 
1997: Family Life and Family Policies in Europe. Oxford: Clarendon Press.

Kilkey, Majella und Jonathan Bradshaw, 1997: Lone mothers, economic well-being, and policies S. 147-184, in: Diane Sainsbury (Hg.), Gender and Welfare State Regimes. Oxford: Oxford University Press.

Klein, Thomas, 1999: Pluralisierung versus Umstrukturierung am Beispiel partnerschaftlicher Lebensformen. Kölner Zeitschrift für Soziologie und Sozialpsychologie 51: 469-490.

Köhler, Sabine, Bernhard Schimpl-Neimanns und Norbert Schwarz, 2000: Pilotprojekt zur Erleichterung der Nutzungsmöglichkeiten von faktisch anonymisierten Mikrodaten. Wirtschaft und Statistik, Heft 1: 30-37.

Konietzka, Dirk und Michaela Kreyenfeld, 2002a: Women's employment and non-marital childbearing - A comparison between East and West Germany in the 1990s. Population 57 (English edition): 331-357.

Konietzka, Dirk und Michaela Kreyenfeld, 2002b: Travail féminin et fécondité hors mariage en Allemagne au cours des années 1990: Comparaison entre l'Est et l'Ouest. Population 57 (édition française): 359-388.

Kreyenfeld, Michaela, 2003: Crisis or adaptation reconsidered: A comparison of East and West German fertility in the first six years after the 'Wende'. European Journal of Population 19: 303-329.

Kreyenfeld, Michaela und Dirk Konietzka, 2003: Neue Familienformen im konservativen Wohlfahrtsstaat? Heirat, Familiengründung und Lebensformen in Ost- und Westdeutschland: Eine Analyse der Mikrozensen 1996 und 2000. Vortrag auf der 3. Mikrozensus-Nutzerkonferenz, Mannheim, Oktober 2003 (www.gesis.org/Dauerbeobachtung/Mikrodaten/Veranstaltungen/3Nutzerkonferenz_beitraege.htm).

Kreyenfeld, Michaela und Dirk Konietzka, 2004: Anpassung oder Verfestigung von Differenzen? Geburtenentwicklung und Familienformen in Ost- und Westdeutschland. MPIDR Working Paper 2004-025: Max-Planck-Institut für demografische Forschung (www.demogr.mpg.de/publications/working.htm).

Lewis, Jane, 1992: Gender and the development of welfare regimes. Journal of European Social Policy 2: 159-173. 
Lewis, Jane, 1997: Lone Mothers in European Welfare Regimes: Shifting Policy Logic. London: Jessica Kingsley Publishers.

Lichter, Daniel T. und Martha L. Crowley, 2004: Welfare reform and child poverty: effects of maternal employment, marriage and cohabitation. Social Science Research 33: 385-408.

Lichter, Daniel T. und Rukamalie Jayakody, 2002: Welfare reform: How do we measure success? Annual Review of Sociology 28: 117-141.

Lister, Ruth, 1994: 'She Has Other Duties' - Women, Citizenship, and Social Security. S. 31-44 in: Sally Baldwin und Jane Falkingham (Hg.), Social Security and Social Change: New Challenges to the Beveridge Model. New York: Harvester Wheatsheaf.

Manow, Philip, 2002: ,The Good, the Bad, and the Ugly': Esping-Andersens SozialstaatsTypologie und die konfessionellen Wurzeln des westlichen Wohlfahrtsstaats. Kölner Zeitschrift für Soziologie und Sozialpsychologie 54: 203-225.

McLanahan, Sara, 2000: Family, state and child well-being. Annual Review of Sociology 26: 703-706.

McLaughlin, Diane, 1997: Poverty and the marital behavior of young women. Journal of Marriage and the Family 59: 582-594.

Meyer, Thomas, 1993: Der Monopolverlust der Familie. Vom Teilsystem der Familie zum Teilsystem privater Lebensformen. Kölner Zeitschrift für Soziologie und Sozialpsychologie 45: $23-40$.

Mitterauer, Michael, 1983: Ledige Mütter: Zur Geschichte unehelicher Geburten in Europa. München: Beck.

Moffitt, Robert, 2000: Welfare benefits and female headship in U.S. time series. American Economic Review 90: 373-377.

Moffitt, Robert, Robert Reville und Anne E. Winkler, 1998: Beyond single mothers: Cohabitation and marriage in the AFDC program. Demography 35: 259-287.

Nave-Herz, Rosemarie, 1997: Pluralisierung familialer Lebensformen ein Konstrukt der Wissenschaft. S. 36-49 in: Laszlo. A. Vaskovics (Hg.), Familienleitbilder und 
Familienrealitäten. Opladen: Leske und Budrich.

Orloff, Ann, 1993: Gender and the social rights of citizenship: The comparative analysis of gender relations and welfare states. American Sociological Review 58: 303-328.

Ostner, Ilona, 1995: Arm ohne Ehemann? Sozialpolitische Regulierung von Lebenschancen für Frauen im internationalen Vergleich. Aus Politik und Zeitgeschichte B 36-37: 3-12.

Pfau-Effinger, Birgit, 1998: Der soziologische Mythos von der Hausfrauenehe - soziohistorische Entwicklungspfade der Familie. Soziale Welt 49: 167-182.

Rupp, Marina, 1998: Lebensverhältnisse nichtverheirateter Frauen beim Übergang zur Elternschaft. S. 41-69 in: Walter Bien und Norbert F. Schneider (Hg.), Kind ja, Ehe nein? Opladen.

Sachße, Christoph, 2003: Subsidiarität. Leitmaxime deutscher Wohlfahrtsstaatlichkeit. S. 191212 in: Stephan Lessenich (Hg.), Wohlfahrtsstaatliche Grundbegriffe. Frankfurt/M.: Campus.

Sainsbury, Diane, 1996: Gender, Equality and Welfare States. Cambridge: Cambridge University Press.

Sainsbury, Diane, 1997: Taxation, family responsibilities, and employment. S. 185-209 in: Dies. (Hg.), Gender and Welfare State Regimes. Oxford: Oxford University Press.

Schimpl-Neimanns, Bernhard, 1998: Analysemöglichkeiten des Mikrozensus. ZUMANachrichten 42: 91-122.

Sigle-Rushton, Wendy und Sara McLanahan, 2002: The living arrangements of new unmarrried mothers. Demography 39: 415-433.

Statistisches Bundesamt, 1996: Sozialleistungen: Tageseinrichtungen für Kinder 1994. Fachserie 13, Reihe 6.3.1. Stuttgart: Statistisches Bundesamt.

Statistisches Bundesamt, 2001a: Bevölkerung und Erwerbstätigkeit: Gebiet und Bevölkerung 2000. Stuttgart: Statistisches Bundesamt.

Statistisches Bundesamt, 2001b: Sozialleistungen: Tageseinrichtungen für Kinder 1998. Fachserie 13, Reihe 6.3.1. Stuttgart: Statistisches Bundesamt. 
Statistisches Bundesamt, 2004: Sozialleistungen: Tageseinrichtungen für Kinder am 31.12.2002 (http://www.destatis.de/basis/d/solei/tab_juhilf.htm).

Stauder, Johannes, 2002: Neue Typisierung von Haushalten und Lebensformen für den Mikrozensus, Statistische Analysen und Studien NRW, Heft 5: 17-35.

Strohmeier, Klaus-Peter, 1993: Pluralisierung und Polarisierung der Lebensformen in Deutschland. Aus Politik und Zeitgeschichte B 17/93: 11-22.

Trappe, Heike, 1995: Emanzipation oder Zwang? Frauen in der DDR zwischen Beruf, Familie und Sozialpolitik. Berlin: Akademie Verlag.

Tölke, Angelika und Karsten Hank (Hg.), 2004: Männer - Das „,vernachlässigte“ Geschlecht in der Familienforschung. Wiesbaden: Verlag für Sozialwissenschaften.

Wagner, Michael und Gabriele Franzmann, 2000: Die Pluralisierung der Lebensformen. Zeitschrift für Bevölkerungswissenschaft 25: 151-173.

Wingen, Max, 1997: Familienpolitik: Grundlagen und aktuelle Probleme. Bonn: Bundeszentrale für politische Bildung. 